\title{
Hitchin's connection, Toeplitz operators, and symmetry invariant deformation quantization
}

\author{
Jørgen Ellegaard Andersen ${ }^{1}$
}

\begin{abstract}
We introduce the notion of a rigid family of Kähler structures on a symplectic manifold. We then prove that a Hitchin connection exists for any rigid holomorphic family of Kähler structures on any compact pre-quantizable symplectic manifold which satisfies certain simple topological constraints. Using Toeplitz operators we prove that the Hitchin connection induces a unique formal connection on smooth functions on the symplectic manifold. Parallel transport of this formal connection produces equivalences between the corresponding BerezinToeplitz deformation quantizations. In the cases where the Hitchin connection is projectively flat, the formal connections will be flat and we get a symmetry-invariant formal quantization. If a certain cohomological condition is satisfied a global trivialization of this algebra bundle is constructed. As a corollary we get a symmetry-invariant deformation quantization.

Finally, these results are applied to the moduli space situation in which Hitchin originally constructed his connection. First we get a proof that the Hitchin connection in this case is the same as the connection constructed by Axelrod, Della Pietra, and Witten. Second we obtain in this way a mapping class group invariant formal quantization of the smooth symplectic leaves of the moduli space of flat $\mathrm{SU}(n)$-connections on any compact surface.
\end{abstract}

Mathematics Subject Classification (2010). 53D50, 53D55, 47B35.

Keywords. Geometric quantization, Hitchin connection, Toeplitz operators, deformation quantization, star products, moduli space of flat connections.

\section{Contents}

1 Introduction . . . . . . . . . . . . . . . . . . . . . . . . . . 294

2 The Hitchin connection . . . . . . . . . . . . . . 300

3 Berezin-Toeplitz deformation quantization on compact Kähler manifolds . 309

4 The formal Hitchin connection . . . . . . . . . . . . . . . . . . . 314

5 Formal trivializations and symmetry-invariant $*$-products $\ldots \ldots \ldots . . .318$

6 Applications to moduli space of flat connections . . . . . . . . . . 321

References . . . . . . . . . . . . . . . . . 321

\footnotetext{
${ }^{1}$ Supported in part by the center of excellence grant "Center for quantum geometry of Moduli Spaces" from the Danish National Research Foundation.
} 


\section{Introduction}

In [31] Hitchin introduced a connection over Teichmüller space in the bundle one obtains by applying geometric quantization to the moduli spaces of flat $\mathrm{SU}(n)$-connections. Furthermore Hitchin proved that this connection is projectively flat. Hitchin's construction was motivated by Witten's study of quantum Chern-Simons theory in $(2+1)$-dimensions in [50]. In fact, Witten constructed via path integral techniques a quantization of Chern-Simons theory in $(2+1)$-dimensions and argued in [50] that this produced a TQFT indexed by a compact simple Lie group and an integer level $k$.

Combinatorially this theory was first constructed by Reshetikhin and Turaev using representation theory of $U_{q}(\operatorname{sl}(n, \mathbb{C}))$ at $q=e^{(2 \pi i) /(k+n)}$; see [40] and [41]. Subsequently these TQFT's were constructed using skein theory by Blanchet, Habegger, Masbaum, and Vogel in [20], [21], and [19]. In particular these TQFT's provide projective representations of the mapping class groups. The fact that these representations agree with the representations obtained from the projective action of the mapping class group on the projectively covariant constant sections of the Hitchin connection follows by combining the results of a series of papers. First of all, the work of Laszlo [33] provides an identification of the Hitchin connection with the TUY-connection constructed in the bundle of WZW-conformal blocks over Teichmüller space in [47]. In joint work with Ueno ([8], [9], [10], and [11]), we have given a proof, based mainly on the results of [47], that the TUY-construction of the WZW-conformal field theory after twist by a fractional power of an abelian theory, satisfies all the axioms of a modular functor. Furthermore, we have proved that the full $(2+1)$-dimensional TQFT that results from this is isomorphic to the one constructed by BHMV via skein theory as mentioned above.

From Witten's path integral formulation of these theories, one expects that these TQFT's have asymptotic expansions in the level $k$ of the theory. In [1] we considered this question in the abelian case and described how one should make this question precise in the context of the mapping class group representations. As we described in that paper, the natural asymptotic expansion for these sequences of representations is a mapping class group invariant deformation quantization of the moduli space of flat $U(1)$-connections. In this paper we extend these results to the case of the above mentioned representations and the $\mathrm{SU}(n)$-moduli space (see Theorem 1.14 below). In fact we consider a more general setting, in which we can construct the Hitchin connection, build a formal Hitchin connection and understand its relation to the associated Berezin-Toeplitz deformation quantizations. Let us describe the generalized setting we will consider.

Let $(M, \omega)$ be a compact symplectic manifold. Let $I$ be a family of Kähler structures on $(M, \omega)$ parameterized holomorphically by some complex manifold $\mathcal{T}$. Suppose $V$ is a vector field on $\mathcal{T}$. Then we can differentiate $I$ along $V$ and we denote this derivative $V[I]: \mathcal{T} \rightarrow C^{\infty}\left(M, \operatorname{End}\left(T M_{\mathbb{C}}\right)\right)$. We define $\widetilde{G}(V) \in C^{\infty}\left(M, S^{2}\left(T M_{\mathbb{C}}\right)\right)$ by

$$
V[I]=\widetilde{G}(V) \omega,
$$


and define $G(V) \in C^{\infty}\left(M, S^{2}\left(T_{\sigma}\right)\right)^{1}$ such that

$$
\widetilde{G}(V)=G(V)+\bar{G}(V)
$$

for all real vector fields $V$ on $\mathcal{T}$. We see that $\widetilde{G}$ and $G$ are one-forms on $\mathcal{T}$ with values in $C^{\infty}\left(M, S^{2}\left(T M_{\mathbb{C}}\right)\right)$ and $C^{\infty}\left(M, S^{2}\left(T_{\sigma}\right)\right)$ respectively.

Definition 1.1. We say that a complex family $I$ of Kähler structures on $(M, \omega)$ is rigid if

$$
\bar{\partial}_{\sigma}\left(G(V)_{\sigma}\right)=0
$$

for all vector fields $V$ on $\mathcal{T}$ and all points $\sigma \in \mathcal{T}$.

We observe that this condition is restrictive, but we refer to [5] for constructions of examples.

Assume now that $(M, \omega)$ is prequantizable. That means there exists a Hermitian line bundle $(\mathscr{L},(\cdot, \cdot))$ over $M$ with a compatible connection $\nabla$ such that

$$
F_{\nabla}=\frac{i}{2 \pi} \omega
$$

For every $\sigma \in \mathcal{T}$ we use the notation $M_{\sigma}=\left(M, I_{\sigma}\right)$ and consider the finitedimensional subspace of $C^{\infty}\left(M, \mathscr{L}^{k}\right)$ given by

$$
H_{\sigma}^{(k)}=H^{0}\left(M_{\sigma}, \mathscr{L}^{k}\right)=\left\{s \in C^{\infty}\left(M, \mathscr{L}^{k}\right) \mid \nabla_{\sigma}^{0,1} s=0\right\} .
$$

We will assume that these subspaces of holomorphic sections form a smooth finite rank subbundle $H^{(k)}$ of the trivial bundle $\mathscr{H}^{(k)}=\mathcal{T} \times C^{\infty}\left(M, \mathscr{L}^{k}\right)$. By semicontinuity, restriction to an open dense subset of $\mathcal{T}$, if necessary, will guaranty this assumption. See also Remark 2.5 below.

Definition 1.2. A connection in $\mathscr{H}^{(k)}$ over $\mathcal{T}$ of the form

$$
\hat{\nabla}=\hat{\nabla}^{t}-u
$$

where $\hat{\nabla}^{t}$ is the trivial connection in $\mathscr{H}^{(k)}$ and $u$ is a one form on $\mathcal{T}$ with values in differential operators acting on $C^{\infty}\left(M, \mathscr{L}^{k}\right)$ is called a Hitchin connection if it preserves the subbundle $H^{(k)}$.

We have the following existence result.

Theorem 1.3. Suppose that I is a rigid family of Kähler structures on the symplectic prequantizable compact manifold $(M, \omega)$, which satisfies that there exists $n \in \mathbb{Z}$ such that the first Chern class of $(M, \omega)$ is $n[\omega] \in H^{2}(M, \mathbb{Z})$ and $H^{1}(M, \mathbb{R})=0$. Then

\footnotetext{
${ }^{1}$ We denote the holomorphic tangent bundle of $\left(M, I_{\sigma}\right)$ by $T_{\sigma}$.
} 
there exists a Hitchin connection $\hat{\nabla}$ in the bundle $\mathscr{H}^{(k)}$ which preserves the subbundle $H^{(k)}$. It is given by

$$
\hat{\nabla}_{V}=\hat{\nabla}_{V}^{t}-u(V),
$$

where $\hat{\nabla}^{t}$ is the trivial connection in $\mathscr{H}^{(k)}, V$ is any smooth vector field on $\mathcal{T}$ and $u(V)$ is the second order differential operator given by

$$
u(V)(s)=\frac{1}{2 k+n}\left\{\frac{1}{2} \Delta_{G(V)}(s)-\nabla_{G(V) d F}(s)+2 k V^{\prime}[F] s\right\},
$$

where $\Delta_{G(V)}$ is a certain second order operator depending linearly and smoothly on $V$ defined by eq. (5) below, $V^{\prime}$ denotes the $(1,0)$-part of the vector field $V$ on $\mathcal{T}$ and $F: \mathcal{T} \rightarrow C_{0}^{\infty}(M)$ is determined by $F_{\sigma} \in C_{0}^{\infty}(M)$ being the Ricci potential for $\left(M, I_{\sigma}\right)$ for all $\sigma \in \mathcal{T}$.

We prove this theorem in Section 2. When we apply this theorem to the gauge theory example discussed in Section 6, we get the following corollary.

Theorem 1.4. The Hitchin connection agrees with the connection constructed by Axelrod, Della Pietra, and Witten in [17].

This was widely believed to be the case, but has not been established before.

In the work of Scheinost and Schottenloher [43], further gauge theory examples of the setup in Theorem 1.3 have been provided. We here give a pure differential geometric construction of the Hitchin connection constructed in their work as well.

Remark 1.5. In [5], in joint work with Gammelgaard and Lauridsen, we use halfforms and the metaplectic correction to prove the existence of a Hitchin connection in the context of metaplectic quantization. The assumption that the first Chern class of $(M, \omega)$ is $n[\omega] \in H^{2}(M, \mathbb{Z})$ is then just replaced by the vanishing of the second Stiefel-Whitney class of $M$. Furthermore, [5] also includes the case of non-compact symplectic manifolds $(M, \omega)$. For this to work, the metaplectic correction is not essential as we will explain in the next remark.

Remark 1.6. The approach presented here also proves the existence of a Hitchin connection for non-compact $(M, \omega)$ and rigid families of complex structures on them, which satisfies the same assumptions as in Theorem 1.3. This follows from the remarks made after the proofs of Lemma 2.6, 2.8, and 2.9 in Section 2. We recall that in [5] we establish that there are many examples in the non-compact case of rigid families of complex structures which satisfies all the assumptions of Theorem 1.3.

Suppose $\Gamma$ is a group which acts on $\mathcal{T}$ and on $M$, such that $I$ is $\Gamma$ equivariant. Assume further that there is an action of $\Gamma$ on the prequantum bundle $(\mathscr{L},(\cdot, \cdot), \nabla)$ covering the $\Gamma$-action on $M$. It then follows that Hitchin's connection is $\Gamma$-invariant.

For compact $M, H_{\sigma}^{(k)}$ is a finite-dimensional subspace of $C^{\infty}\left(M, \mathscr{L}^{k}\right)=\mathscr{H}_{\sigma}^{(k)}$ 
and therefore closed, thus we have the orthogonal projection $\pi_{\sigma}^{(k)}: \mathscr{H}_{\sigma}^{(k)} \rightarrow H_{\sigma}^{(k)}$. Since $H^{(k)}$ is a smooth subbundle of $\mathscr{H}^{(k)}$ the projections $\pi_{\sigma}^{(k)}$ form a smooth map $\pi^{(k)}$ from $\mathcal{T}$ to the space of bounded operators on the $L_{2}$-completion of $C^{\infty}\left(M, \mathscr{L}^{k}\right)$.

From these projections we can construct the Toeplitz operators associated to any smooth function $f \in C^{\infty}(M), T_{f, \sigma}^{(k)}: \mathscr{H}_{\sigma}^{(k)} \rightarrow H_{\sigma}^{(k)}$, defined by

$$
T_{f, \sigma}^{(k)}(s)=\pi_{\sigma}^{(k)}(f s)
$$

for any element $s$ in $\mathscr{H}_{\sigma}^{(k)}$ and any point $\sigma \in \mathcal{T}$. We observe that the Toeplitz operators are smooth sections $T_{f}^{(k)}$ of the bundle $\operatorname{Hom}\left(\mathscr{H}^{(k)}, H^{(k)}\right)$ and restricts to smooth sections of $\operatorname{End}\left(H^{(k)}\right)$.

Let $\mathscr{D}(M)$ be the space of smooth differential operators on $M$ acting on smooth functions on $M$. Let $C_{h}$ be the trivial $C^{\infty}(M) \llbracket h \rrbracket$-bundle over $\mathcal{T}$.

Definition 1.7. A formal connection $D$ is a connection in $C_{h}$ over $\mathcal{T}$ of the form

$$
D_{V} f=V[f]+\widetilde{D}(V)(f),
$$

where $\widetilde{D}$ is a smooth one-form on $\mathcal{T}$ with values in $\mathscr{D}_{h}(M)=\mathscr{D}(M) \llbracket h \rrbracket, f$ is any smooth section of $C_{h}, V$ is any smooth vector field on $\mathcal{T}$ and $V[f]$ is the derivative of $f$ in the direction of $V$.

For a formal connection we get the series of differential operators $\widetilde{D}^{(l)}$ given by

$$
\widetilde{D}(V)=\sum_{l=0}^{\infty} \widetilde{D}^{(l)}(V) h^{l} .
$$

From Hitchin's connection in $H^{(k)}$ we get an induced connection $\hat{\nabla}^{e}$ in the endomorphism bundle $\operatorname{End}\left(H^{(k)}\right)$. The Toeplitz operators are not covariant constant sections with respect to $\hat{\nabla}^{e}$. They are asymptotically covariant constant in $k$ in the following very precise sense.

Theorem 1.8. Under the same assumptions as in Theorem 1.3, there is a unique formal Hitchin connection $D$ which satisfies that

$$
\hat{\nabla}_{V}^{e} T_{f}^{(k)} \sim T_{\left(D_{V} f\right)(1 /(2 k+n))}^{(k)}
$$

for all smooth sections $f$ of $C_{h}$ and all smooth vector fields on $\mathcal{T}$. Moreover

$$
\widetilde{D}=0 \bmod h \text {. }
$$

Here $\sim$ means the following: for all $L \in \mathbb{Z}_{+}$we have that

$$
\left\|\hat{\nabla}_{V}^{e} T_{f}^{(k)}-\left(T_{V[f]}^{(k)}+\sum_{l=1}^{L} T_{\tilde{D}_{V}^{(l)} f}^{(k)} \frac{1}{(2 k+n)^{l}}\right)\right\|=O\left(k^{-(L+1)}\right)
$$

uniformly over compact subsets of $\mathcal{T}$ for all smooth maps $f: \mathcal{T} \rightarrow C^{\infty}(M)$. 
This theorem is proved in Section 4. For an explicit formula for $\widetilde{D}$ see (19).

Again in the presence of a symmetry group $\Gamma$ as before, the formal Hitchin connection becomes $\Gamma$-invariant.

By the work of Bordeman, Meinrenken, and Schlichenmaier, [22], [44], [45], and [46], applied to the Kähler manifold $\left(M, \omega, I_{\sigma}\right)$, we know that for any $f, g \in$ $C^{\infty}(M)$, there is an asymptotic expansion

$$
T_{f, \sigma}^{(k)} T_{g, \sigma}^{(k)} \sim \sum_{l=0}^{\infty} T_{c_{\sigma}^{(l)}(f, g), \sigma}^{(k)} k^{-l},
$$

where $c_{\sigma}^{(l)}(f, g) \in C^{\infty}(M)$ are uniquely determined. Moreover it gives a deformation quantization

$$
f \star_{\sigma}^{B T} g=\sum_{l=0}^{\infty} c_{\sigma}^{(l)}(f, g) h^{l}
$$

which is known as the Berezin-Toeplitz quantization. By the work of Karabegov and Schlichenmaier [32], it is known to be a differential deformation quantization.

Proposition 1.9. For every vector field $V$ on $\mathcal{T}$, the formal operator $D_{V}$ constructed in Theorem 1.8 is a derivation for $\star_{\sigma}^{B T}$.

Let $\mathcal{A}_{h}$ be the vector space of sections of $C_{h}$ which are covariant constant with respect to the formal Hitchin connection $D$ over $\mathcal{T}$. Then by Proposition 1.9, we see that the star products $\star_{\sigma}^{B T}, \sigma \in \mathcal{T}$, induces an associative algebra structure on $\mathcal{A}_{h}$. Moreover, the symmetry group $\Gamma$ will act by automorphisms of $\mathcal{A}_{h}$.

Theorem 1.10. If the formal Hitchin connection has trivial global holonomy over $\mathcal{T}$, then the algebra $\mathcal{A}_{h}$ is a formal quantization of the Poisson algebra of smooth functions on $(M, \omega)$.

Remark 1.11. If the Hitchin connection is projectively flat and $\mathcal{T}$ is simply connected, then the formal Hitchin connection has trivial global holonomy over $\mathcal{T}$.

Let $C_{h}^{\infty}(M)=C^{\infty}(M) \llbracket h \rrbracket$. A formal trivialization of a formal connections is defined as follows.

Definition 1.12. A formal trivialization of a formal connection $D$ is a smooth map $P: \mathcal{T} \rightarrow \mathscr{D}_{h}(M)$ which modulo $h$ is an isomorphism for all $\sigma \in \mathcal{T}$ and such that

$$
D_{V}(P(f))=0
$$

for all vector fields $V$ on $\mathcal{T}$ and all $f \in C_{h}^{\infty}(M)$. 
The existence of a formal trivialization is of course equivalent to the triviality of the global holonomy of $D$ over $\mathcal{T}$. In Section 5 we construct a global $\Gamma$-equivariant formal trivialization under the assumption that the Hitchin connection is projectively flat and that the first $\Gamma$-equivariant cohomology of $M$ with coefficients in the $\Gamma$-module consisting of all differential operators on $M$ vanishes. This further leads to the construction of a $\Gamma$ invariant $*$-product on $M$, simply because a global trivialization induces a vector space isomorphism between $\mathcal{A}_{h}$ and $C_{h}^{\infty}(M)$.

Theorem 1.13. Assume that the formal Hitchin connection D is flat and

$$
H_{\Gamma}^{1}(\mathcal{T}, D(M))=0,
$$

then there is a $\Gamma$-invariant trivialization $P$ of $D$ and the *-product

$$
f \star g=P_{\sigma}^{-1}\left(P_{\sigma}(f) \star_{\sigma}^{B T} P_{\sigma}(g)\right)
$$

is independent of $\sigma \in \mathcal{T}$ and $\Gamma$-invariant.

We apply our results to the moduli space of flat connections on a surface. Let $\Sigma$ be a compact 2-dimensional manifold and $\mathcal{M}$ the moduli space of flat $\mathrm{SU}(n)$-connections on $\Sigma$. Let $\Gamma$ be the mapping class group of $\Sigma$. The $\Gamma$ action on $\mathcal{M}$ is Poisson and it preserves all the symplectic leaves of $\mathcal{M}$. Let $\mathcal{T}$ be Teichmüller space of $\Sigma$. Then for each smooth symplectic leaf $(M, \omega)$ of $\mathcal{M}$ we have a holomorphic map $I$ from $\mathcal{T}$ to the space of complex structures on $(M, \omega)$ which is $\Gamma$-equivariant.

Applying the above to this moduli space situation, we get the following theorem.

Theorem 1.14. There is a mapping class group invariant formal quantization on the smooth symplectic leaves $(M, \omega)$ of the moduli space of flat $\mathrm{SU}(n)$-connections on any compact surface.

It seems a very interesting open problem to understand how this formal quantization is related to the quantization of the moduli spaces presented in [6] and [7], as we have done in the abelian case in [1].

We remark that the interplay between Toeplitz operators and Hitchin's connection forms the foundation for the proof of the asymptotic faithfulness theorem in [2], the determination of the Nielsen-Thurston types of mapping classes via TQFT [3] and forms our example of a representation of the mapping class group, which has no fixed vectors, but which has an almost fixed vector, i.e. proving the mapping class groups do not have Kazhdan's property (T); see [4]. 


\section{The Hitchin connection}

Let $(M, \omega)$ be a compact symplectic manifold.

Definition 2.1. A prequantum line bundle $(\mathscr{L},(\cdot, \cdot), \nabla)$ over the symplectic manifold $(M, \omega)$ consist of a complex line bundle $\mathscr{L}$ with a Hermitian structure $(\cdot, \cdot)$ and a compatible connection $\nabla$ whose curvature is

$$
F_{\nabla}=\frac{i}{2 \pi} \omega,
$$

e.g.

$$
\nabla_{X} \nabla_{Y}-\nabla_{Y} \nabla_{X}-\nabla_{[X, Y]}=\omega(X, Y)
$$

for all vector fields $X, Y$ on $M$. We say that the symplectic manifold $(M, \omega)$ is prequantizable if there exists a prequantum line bundle over it.

Recall that the condition for the existence of a prequantum line bundle is that $[\omega] \in \operatorname{Im}\left(H^{2}(M, \mathbb{Z}) \rightarrow H^{2}(M, \mathbb{R})\right)$ and that the inequivalent choices of prequantum line bundles (if they exists) are parameterized by $H^{1}(M, U(1))$; see e.g. [51].

We shall assume that $(M, \omega)$ is prequantizable and fix a prequantum line bundle $(\mathscr{L},(\cdot, \cdot), \nabla)$ over $(M, \omega)$.

Assume that $\mathcal{T}$ is a smooth manifold which smoothly parametrizes Kähler structures on $(M, \omega)$. This means we have a $\operatorname{smooth}^{2}$ map $I: \mathcal{T} \rightarrow C^{\infty}(M, \operatorname{End}(T M))$ such that $\left(M, \omega, I_{\sigma}\right)$ is a Kähler manifold for each $\sigma \in \mathcal{T}$.

We will use the notation $M_{\sigma}$ for the complex manifold $\left(M, I_{\sigma}\right)$. For each $\sigma \in \mathcal{T}$ we use $I_{\sigma}$ to split the complexified tangent bundle $T M_{\mathbb{C}}$ into the holomorphic and the anti-holomorphic parts, which we denote

$$
T_{\sigma}=E\left(I_{\sigma}, i\right)=\operatorname{Im}\left(\mathrm{Id}-i I_{\sigma}\right)
$$

and

$$
\bar{T}_{\sigma}=E\left(I_{\sigma},-i\right)=\operatorname{Im}\left(\operatorname{Id}+i I_{\sigma}\right)
$$

respectively.

The real Kähler-metric $g_{\sigma}$ on $\left(M_{\sigma}, \omega\right)$ extended complex linearly to $T M_{\mathbb{C}}$ is by definition

$$
g_{\sigma}(X, Y)=\omega\left(X, I_{\sigma} Y\right),
$$

where $X, Y \in C^{\infty}(M, T M \otimes \mathbb{C})$. Both $g_{\sigma}$ and $\omega$ induce isomorphisms

$$
i_{g_{\sigma}}, i_{\omega}: T M_{\mathbb{C}} \rightarrow T^{*} M_{\mathbb{C}}
$$

\footnotetext{
${ }^{2}$ Here a smooth map from $\mathcal{T}$ to $C^{\infty}(M, W)$ for any smooth vector bundle $W$ over $M$ means a smooth section of $\pi_{M}^{*}(W)$ over $\mathcal{T} \times M$, where $\pi_{M}$ is the projection onto $M$. Likewise a smooth $p$-form on $\mathcal{T}$ with values in $C^{\infty}(M, W)$ is by definition a smooth section of $\pi_{\mathcal{T}}^{*} \Lambda^{p}(\mathcal{T}) \otimes \pi_{M}^{*}(W)$ over $\mathcal{T} \times M$. We will also encounter the situation where we have a bundle $\tilde{W}$ over $\mathcal{T} \times M$ and then we will talk about a smooth $p$-form on $\mathcal{T}$ with values in $C^{\infty}\left(M, \widetilde{W}_{\sigma}\right)$ and mean a smooth section of $\pi_{\mathcal{T}}^{*} \Lambda^{p}(\mathcal{T}) \otimes \widetilde{W}$ over $\mathcal{T} \times M$.
} 
and they are related by

$$
i_{g_{\sigma}}=-I_{\sigma} i_{\omega}
$$

We record for later use that since $\Lambda^{2}\left(I_{\sigma}\right) \omega=\omega$, we get that

$$
\left(\Lambda^{2} i_{g_{\sigma}}\right)^{-1}(\omega)=\left(\Lambda^{2} i_{\omega}\right)^{-1}(\omega) \text {. }
$$

Suppose $V$ is a vector field on $\mathcal{T}$. Then we can differentiate $I$ along $V$ and we denote this derivative $V[I]: \mathcal{T} \rightarrow C^{\infty}\left(M, \operatorname{End}\left(T M_{\mathbb{C}}\right)\right)$. Differentiating the equation $I^{2}=-\mathrm{Id}$, we see that $V[I]$ anti-commutes with $I$. Hence we get that

$$
V[I]_{\sigma} \in C^{\infty}\left(M,\left(T_{\sigma}^{*} \otimes \bar{T}_{\sigma}\right) \oplus\left(\bar{T}_{\sigma}^{*} \otimes T_{\sigma}\right)\right)
$$

for each $\sigma \in \mathcal{T}$. Let

$$
V[I]_{\sigma}=V[I]_{\sigma}^{\prime \prime}+V[I]_{\sigma}^{\prime}
$$

be the corresponding decomposition such that $V[I]_{\sigma}^{\prime \prime} \in C^{\infty}\left(M, T_{\sigma}^{*} \otimes \bar{T}_{\sigma}\right)$ and $V[I]_{\sigma}^{\prime} \in C^{\infty}\left(M, \bar{T}_{\sigma}^{*} \otimes T_{\sigma}\right)$.

Now we will further assume that $\mathcal{T}$ is a complex manifold and that $I$ is a holomorphic map from $\mathcal{T}$ to the space of all complex structures on $M$. Concretely, this means that

$$
V^{\prime}[I]_{\sigma}=V[I]_{\sigma}^{\prime}
$$

and

$$
V^{\prime \prime}[I]_{\sigma}=V[I]_{\sigma}^{\prime \prime}
$$

for all $\sigma \in \mathcal{T}$, where $V^{\prime}$ means the $(1,0)$-part of $V$ and $V^{\prime \prime}$ means the $(0,1)$-part of $V$ over $\mathcal{T}$.

We see that

$$
V[g](X, Y)=\omega(X, V[I] Y) .
$$

Since $\omega$ is of type $(1,1)$ and $g$ is symmetric, we see that

$$
V[g] \in C^{\infty}\left(M, S^{2}\left(T^{*}\right) \oplus S^{2}\left(\bar{T}^{*}\right)\right)
$$

and self-conjugate for real vector fields. Let us now define

$$
\widetilde{G}(V) \in C^{\infty}\left(M, T M_{\mathbb{C}} \otimes T M_{\mathbb{C}}\right)
$$

by

$$
V[I]=\widetilde{G}(V) \omega,
$$

where the notation $\widetilde{G}(V) \omega$ means contraction of tangent and cotangent vectors and define $G(V) \in C^{\infty}\left(M, T_{\sigma} \otimes T_{\sigma}\right)$ such that

$$
\widetilde{G}(V)=G(V)+\bar{G}(V)
$$

for all real vector fields $V$ on $\mathcal{T}$. We see that $\widetilde{G}$ and $G$ are one-forms on $\mathcal{T}$ with values in $C^{\infty}\left(M, T M_{\mathbb{C}} \otimes T M_{\mathbb{C}}\right)$ and $C^{\infty}\left(M, T_{\sigma} \otimes T_{\sigma}\right)$ respectively. We observe that

$$
V^{\prime}[I]=G(V) \omega
$$


and $G(V)=G\left(V^{\prime}\right)$.

Since $V[g]=\omega V[I]$, we see that

$$
V[g]=\omega \widetilde{G}(V) \omega=i_{\omega} \otimes i_{\omega}(\widetilde{G}(V)) .
$$

From this it is clear that $\widetilde{G}$ takes values in $C^{\infty}\left(M, S^{2}\left(T M_{\mathbb{C}}\right)\right)$ and therefore that $G$ takes values in $C^{\infty}\left(M, S^{2}\left(T_{\sigma}\right)\right)$.

On $\mathscr{L}^{k}$ we have the smooth family of $\bar{\partial}$-operators $\nabla^{0,1}$ defined at $\sigma \in \mathcal{T}$ by

$$
\nabla_{\sigma}^{0,1}=\frac{1}{2}\left(1+i I_{\sigma}\right) \nabla .
$$

For every $\sigma \in \mathcal{T}$ we consider the finite-dimensional subspace of $C^{\infty}\left(M, \mathscr{L}^{k}\right)$ given by

$$
H_{\sigma}^{(k)}=H^{0}\left(M_{\sigma}, \mathscr{L}^{k}\right)=\left\{s \in C^{\infty}\left(M, \mathscr{L}^{k}\right) \mid \nabla_{\sigma}^{0,1} s=0\right\} .
$$

We will assume that these subspaces of holomorphic sections form a smooth finite rank subbundle $H^{(k)}$ of the trivial bundle $\mathscr{H}^{(k)}=\mathcal{T} \times C^{\infty}\left(M, \mathscr{L}^{k}\right)$. See also the remark regarding this in the introduction and also the Remark 2.5 below.

Let $\hat{\nabla}^{t}$ denote the trivial connection in the trivial bundle $\mathcal{T} \times C^{\infty}\left(M, \mathscr{L}^{k}\right)$. Let $\mathscr{D}\left(M, \mathscr{L}^{k}\right)$ denote the vector space of differential operators on $C^{\infty}\left(M, \mathscr{L}^{k}\right)$. For any smooth one-form $u$ on $\mathcal{T}$ with values in $\mathscr{D}\left(M, \mathscr{L}^{k}\right)$ we have a connection $\hat{\nabla}$ in $\mathscr{H}^{(k)}$ given by

$$
\hat{\nabla}_{V}=\hat{\nabla}_{V}^{t}-u(V)
$$

for any vector field $V$ on $\mathcal{T}$.

Lemma 2.2. The connection $\hat{\nabla}$ in $\mathscr{H}^{(k)}$ induces a connection in $H^{(k)}$, i.e. $\hat{\nabla}$ is a Hitchin connection as defined in Definition 1.2, if and only if

$$
\frac{i}{2} V[I] \nabla^{1,0} s+\nabla^{0,1} u(V) s=0
$$

for all vector fields $V$ on $\mathcal{T}$ and all smooth sections s of $H^{(k)}$.

Proof. Let $s$ be a section of $H^{(k)}$ over $\mathcal{T}$ and $V$ a vector field on $\mathcal{T}$. Then $\hat{\nabla}_{V} s$ is a section of $\mathscr{H}^{(k)}$, and we compute at a point $\sigma \in \mathcal{T}$ that

$$
\begin{aligned}
\nabla_{\sigma}^{0,1}\left(\left(\hat{\nabla}_{V}(s)\right)_{\sigma}\right) & =\nabla_{\sigma}^{0,1}\left(V[s]_{\sigma}\right)-\nabla_{\sigma}^{0,1}\left((u(V) s)_{\sigma}\right) \\
& =-\frac{i}{2}\left(V[I] \nabla^{1,0} s\right)_{\sigma}-\nabla_{\sigma}^{0,1}\left((u(V) s)_{\sigma}\right),
\end{aligned}
$$

since

$$
\frac{i}{2}\left(V[I] \nabla^{1,0} s\right)_{\sigma}+\nabla_{\sigma}^{0,1}\left(V[s]_{\sigma}\right)=0 .
$$

Hence $\hat{\nabla}$ preserves $H^{(k)}$ if and only if (4) holds. 
We observe that

$$
V^{\prime \prime}[I] \nabla^{1,0} s=0,
$$

so $u\left(V^{\prime \prime}\right)=0$ solves (4) along the anti-holomorphic directions on $\mathcal{T}$. In other words the $(0,1)$-part of the trivial connection $\hat{\nabla}^{t}$ induces a $\bar{\partial}$-operator on $H^{(k)}$ and hence makes it a holomorphic vector bundle over $\mathcal{T}$.

This is of course not in general the situation in the $(1,0)$ direction. Let us now consider a particular $u$ and prove that it solves (4) under certain conditions.

On the Kähler manifold $\left(M_{\sigma}, \omega\right)$ we have the Kähler metric and we have the Levi-Civita connection $\nabla$ in $T_{\sigma}$. We also have the Ricci potential $F_{\sigma} \in C_{0}^{\infty}(M, \mathbb{R})$. Here

$$
C_{0}^{\infty}(M, \mathbb{R})=\left\{f \in C^{\infty}(M, \mathbb{R}) \mid \int_{M} f \omega^{m}=0\right\}
$$

and the Ricci potential is the element of $C_{0}^{\infty}(M, \mathbb{R})$ which satisfies

$$
\operatorname{Ric}_{\sigma}=\operatorname{Ric}_{\sigma}^{H}+2 i \partial_{\sigma} \bar{\partial}_{\sigma} F_{\sigma},
$$

where $\operatorname{Ric}_{\sigma} \in \Omega^{1,1}\left(M_{\sigma}\right)$ is the Ricci form and $\operatorname{Ric}_{\sigma}^{H}$ is its harmonic part. We see that we get this way a smooth function $F: \mathcal{T} \rightarrow C_{0}^{\infty}(M, \mathbb{R})$.

For any $G \in C^{\infty}\left(M, S^{2}\left(T_{\sigma}\right)\right)$ we get a linear bundle map

$$
G: T_{\sigma}^{*} \rightarrow T_{\sigma}
$$

and we have the operator

$$
\begin{aligned}
\Delta_{G}: C^{\infty} & \left(M, \mathscr{L}^{k}\right) \stackrel{\nabla_{\sigma}^{1,0}}{\longrightarrow} C^{\infty}\left(M, T_{\sigma}^{*} \otimes \mathscr{L}^{k}\right) \stackrel{G \otimes \mathrm{Id}}{\longrightarrow} C^{\infty}\left(M, T_{\sigma} \otimes \mathscr{L}^{k}\right) \\
& \stackrel{\nabla_{\sigma}^{1,0} \otimes \operatorname{Id}+\operatorname{Id} \otimes \nabla_{\sigma}^{1,0}}{\longrightarrow} C^{\infty}\left(M, T_{\sigma}^{*} \otimes T_{\sigma} \otimes \mathscr{L}^{k}\right) \stackrel{\operatorname{Tr}}{\longrightarrow} C^{\infty}\left(M, \mathscr{L}^{k}\right) .
\end{aligned}
$$

For any smooth function $f$ on $M$, we get a vector field

$$
G d f \in C^{\infty}\left(M, T_{\sigma}\right) .
$$

Implicit in this definition is the projection from $T M \cong T_{\sigma} \oplus \bar{T}_{\sigma}$ to $T_{\sigma}$, which takes $d f$ to $\partial_{\sigma} f$.

Putting these constructions together we consider the following operator for some $n \in \mathbb{Z}$ such that $2 k+n \neq 0$

$$
u(V)=\frac{1}{2 k+n} o(V)+V^{\prime}[F]
$$

where

$$
o(V)=\frac{1}{2} \Delta_{G(V)}-\nabla_{G(V) d F}-n V^{\prime}[F] .
$$

The connection associated to this $u$ is denoted $\hat{\nabla}$ and we call it the Hitchin connection. 
Definition 2.3. We say that the complex family $I$ of Kähler structures on $(M, \omega)$ is rigid if

$$
\bar{\partial}_{\sigma}\left(G(V)_{\sigma}\right)=0
$$

for all vector fields $V$ on $\mathcal{T}$ and all points $\sigma \in \mathcal{T}$.

We will assume our holomorphic family $I$ is rigid.

Theorem 2.4. Suppose that I is a rigid family of Kähler structures on the compact symplectic prequantizable manifold $(M, \omega)$, which satisfies that there exists $n \in \mathbb{Z}$ such that the first Chern class of $(M, \omega)$ is $n[\omega] \in H^{2}(M, \mathbb{Z})$ and $H^{1}(M, \mathbb{R})=0$. Then $u$ given by (6) and (7) satisfies (4) for all $k$ such that $2 k+n \neq 0$.

Hence the Hitchin connection $\hat{\nabla}$ preserves the subbundle $H^{(k)}$ under the stated conditions and we have obtained Theorem 1.3.

Remark 2.5. In fact, suppose we don't know off hand that the subspaces $H_{\sigma}^{(k)}, \sigma \in \mathcal{T}$ form a sub-bundle of $\mathscr{H}^{(k)}$, but that we just have a $u$, that satisfies (4). Then this $u$ will induce a connection in $\mathscr{H}^{(k)}$, whose parallel transport will map the subspaces $H_{\sigma}^{(k)}, \sigma \in \mathcal{T}$ to each other. But then parallel transport gives a local trivialization of these subspaces and hence shows that we do in fact have a sub-bundle $H^{(k)}$ of $\mathscr{H}^{(k)}$.

Theorem 2.4 is established through the following three lemmas.

Lemma 2.6. Assume that the first Chern class of $(M, \omega)$ is $n[\omega] \in H^{2}(M, \mathbb{Z})$. For any $\sigma \in \mathcal{T}$ and for any $G \in H^{0}\left(M_{\sigma}, S^{2}\left(T_{\sigma}\right)\right)$ we have the following formula

$$
\begin{aligned}
\nabla_{\sigma}^{0,1}\left(\Delta_{G}(s)-2 \nabla_{G d F_{\sigma}}(s)\right)= & -i(2 k+n) G \omega \nabla_{\sigma}^{1,0}(s) \\
& -i k \operatorname{Tr}\left(-2 G \partial_{\sigma} F \omega+\nabla_{\sigma}^{1,0}(G) \omega\right) s,
\end{aligned}
$$

for all $s \in H^{0}\left(M_{\sigma}, \mathscr{L}^{k}\right)$.

Proof. We compute that

$$
\begin{aligned}
\nabla_{\sigma}^{0,1} & \left(\Delta_{G}(s)\right) \\
& =\operatorname{Tr}\left(\nabla^{0,1} \nabla^{1,0} G \nabla^{1,0}(s)\right) \\
& =\operatorname{Tr}\left(\nabla^{1,0} \nabla^{0,1} G \nabla^{1,0}(s)\right)-i k \operatorname{Tr}\left(\omega G \nabla^{1,0}(s)\right)-i \operatorname{Tr}\left(\operatorname{Ric}_{\sigma} G \nabla^{1,0}(s)\right) \\
& =-i k \operatorname{Tr}\left(\omega G \nabla^{1,0}(s)\right)-i \operatorname{Tr}\left(\operatorname{Ric}_{\sigma} G \nabla^{1,0}(s)\right)-i k \operatorname{Tr}\left(\nabla^{1,0}(G \omega s)\right) \\
& =-2 i k \operatorname{Tr}\left(\omega G \nabla^{1,0}(s)\right)-i \operatorname{Tr}\left(\operatorname{Ric}_{\sigma} G \nabla^{1,0}(s)\right)-i k \operatorname{Tr}\left(\nabla^{1,0}(G) \omega\right) \otimes s,
\end{aligned}
$$


Hitchin's connection, Toeplitz operators, and invariant deformation quantization 305 since $G$ is holomorphic and $\nabla(\omega)=0$ because $\left(M_{\sigma}, \omega\right)$ is Kähler. The assumption $c_{1}(M, \omega)=n[\omega]$ implies that $\operatorname{Ric}_{\sigma}^{H}=n \omega$ and so

$$
\operatorname{Ric}_{\sigma}=n \omega+2 i \partial_{\sigma} \bar{\partial}_{\sigma} F_{\sigma}
$$

From this we conclude the stated formula.

Remark 2.7. The proof of Lemma 2.6 really uses that $M$ is compact, since it is using Hodge theory as stated. However, based on the assumption $c_{1}(M, \omega)=n[\omega]$, we can still define $F_{\sigma}$ such that it satisfies that

$$
\operatorname{Ric}_{\sigma}=n \omega+2 i d \bar{\partial} F_{\sigma}
$$

for all $\sigma \in \mathcal{T}$, even though $M$ is not compact. This follows since any exact $(1,1)$-form on a complex manifold is $\partial \bar{\partial}$-exact. The rest of the proof is a purely local calculation and therefore we do not need that $M$ is compact for either the statement or the proof of Lemma 2.6.

Lemma 2.8. We have the following relation

$$
2 i \bar{\partial}_{\sigma}\left(V^{\prime}[F]_{\sigma}\right)=\frac{1}{2} \operatorname{Tr}\left(2 G(V) \partial(F) \omega-\nabla^{1,0}(G(V)) \omega\right)_{\sigma}
$$

provided $H^{1}(M, \mathbb{R})=0$.

To prove this lemma, we need a formula for the variation of the Ricci form.

Lemma 2.9. For any smooth vector field $V$ on $\mathcal{T}$ we have that

$$
\left(V^{\prime}[\mathrm{Ric}]\right)^{1,1}=-\frac{1}{2} \partial \operatorname{Tr}\left(\nabla^{1,0}(G(V)) \omega\right) .
$$

Proof. The Ricci form $\operatorname{Ric}_{\sigma}$ of the Kähler manifold $\left(M_{\sigma}, \omega\right)$ is by definition

$$
\operatorname{Ric}_{\sigma}=R_{\sigma}(\omega),
$$

where $R_{\sigma} \in C^{\infty}\left(M, S^{2}\left(\Lambda_{\sigma}^{1,1} M\right)\right)$ is the Kähler curvature and

$$
R_{\sigma}(\omega)=R_{\sigma}\left(\left(\Lambda^{2} i_{g_{\sigma}}\right)^{-1}(\omega)\right)
$$

From this we conclude that

$$
V^{\prime}[\mathrm{Ric}]=V^{\prime}[R]\left(\left(\Lambda^{2} i_{\omega}\right)^{-1}(\omega)\right)
$$

where we have used (3). According to Theorem 1.174(c) in [18], we have for any four vector fields $X, Y, Z, U$ on $M$, that

$$
\begin{aligned}
V^{\prime}[R](X, Y, Z, U)=\frac{1}{2} & \left(\nabla_{Y, Z}^{2}\left(V^{\prime}[g]\right)(X, U)+\nabla_{X, U}^{2}\left(V^{\prime}[g]\right)(Y, Z)\right. \\
& -\nabla_{X, Z}^{2}\left(V^{\prime}[g]\right)(Y, U)-\nabla_{Y, U}^{2}\left(V^{\prime}[g]\right)(X, Z) \\
& \left.+V^{\prime}[g](R(X, Y) Z, U)-V^{\prime}[g](R(X, Y) U, Z)\right),
\end{aligned}
$$


where $R(X, Y) \in C^{\infty}(M, \operatorname{End}(T M))$ is $(3,1)$-curvature of $\nabla$, the Levi-Civita connection on $\left(M_{\sigma}, \omega\right)$ and $\nabla_{X, Y}^{2}=\nabla_{X} \nabla_{Y}-\nabla_{\nabla_{X} Y}$. The Levi-Civita connection $\nabla$ of $\left(M_{\sigma}, \omega\right)$ and therefore also $R(X, Y)_{\sigma}$ preserves the type decomposition of tensors on $\left(M_{\sigma}, \omega\right)$. Hence when we want to compute $V^{\prime}[R]$ applied to $\left(\Lambda^{2} i_{\omega}\right)^{-1}(\omega) \in C^{\infty}\left(M, T_{\sigma} \wedge \bar{T}_{\sigma}\right)$, and we are only interested in the $(1,1)$ part of the result, we only get contributions from the third and fourth term. These two terms give

$$
\begin{aligned}
\left(V^{\prime}[\text { Ric }]\right)^{1,1}=- & \frac{1}{2}\left(\operatorname{Tr}\left(\nabla^{1,0} \nabla^{1,0}\left(V^{\prime}[g]\right) \otimes\left(\Lambda^{2} i_{\omega}\right)^{-1}(\omega)\right)\right. \\
& \left.+\operatorname{Tr}\left(\nabla^{0,1} \nabla^{0,1}\left(V^{\prime}[g]\right) \otimes\left(\Lambda^{2} i_{\omega}\right)^{-1}(\omega)\right)\right) .
\end{aligned}
$$

Using that $\nabla(\omega)=0$ and that $V^{\prime}[g]=i_{\omega} \otimes i_{\omega} G(V)$ we get that

$$
\left(V^{\prime}[\text { Ric }]\right)^{1,1}=-\frac{1}{2} \partial \operatorname{Tr}\left(\nabla^{1,0}(G(V)) \otimes \omega\right) .
$$

Remark 2.10. We observe that the proof is a purely local calculation and therefore we do not need that $M$ is compact for either the statement or the proof of Lemma 2.9.

Proof of Lemma 2.8. By the definition of the Ricci potential

$$
\mathrm{Ric}=\operatorname{Ric}^{H}+2 i d \bar{\partial} F
$$

where $\operatorname{Ric}^{H}=n \omega$ by the assumption. Hence

$$
V^{\prime}[\mathrm{Ric}]=-d V^{\prime}[I] d F+2 i d \bar{\partial} V^{\prime}[F]
$$

and therefore

$$
2 i \partial \bar{\partial} V^{\prime}[F]=\left(V^{\prime}[\text { Ric }]\right)^{1,1}+\partial V^{\prime}[I] \partial F .
$$

From the above we conclude that

$$
\frac{1}{2} \operatorname{Tr}\left(2 G(V) \partial F \omega-\nabla^{1,0}(G(V)) \omega\right)_{\sigma}-2 i \bar{\partial}_{\sigma} V^{\prime}[F]_{\sigma} \in \Omega_{\sigma}^{0,1}(M)
$$

is $\partial_{\sigma}$-closed. By Lemma 2.6 it is also $\bar{\partial}_{\sigma}$-closed, hence it is a closed one form on $M$. But since we assume that $H^{1}(M, \mathbb{R})=0$, we see it is exact, but then it in fact vanishes since it is also of type $(0,1)$ on $M_{\sigma}$.

From the above we conclude that

$$
u(V)=\frac{1}{2 k+n}\left\{\frac{1}{2} \Delta_{G(V)}-\nabla_{G(V) d F}+2 k V^{\prime}[F]\right\}
$$

solves (4) and hence we have established Theorem 2.4. 
Remark 2.11. The proof of Lemma 2.8 again really uses that $M$ is compact, since it is using Hodge theory as stated. However we still get $F$ defined as in the remark above following Lemma 2.6.

The following part of the proof also applies in the for non-compact $M$, proving that

$$
\frac{1}{2} \operatorname{Tr}\left(2 G(V) \partial F \omega-\nabla^{1,0}(G(V)) \omega\right)_{\sigma}-2 i \bar{\partial}_{\sigma} V^{\prime}[F]_{\sigma} \in \Omega_{\sigma}^{0,1}(M)
$$

is a closed one form. But then the assumption that $H^{1}(M, \mathbb{R})=0$ implies that there exists a one-form $\beta$ on $\mathcal{T}$ with values in smooth functions on $M$, such that

$$
\bar{\partial} \beta_{\sigma}(V)=\frac{1}{2} \operatorname{Tr}\left(2 G(V) \partial F \omega-\nabla^{1,0}(G(V)) \omega\right)_{\sigma}-2 i \bar{\partial}_{\sigma} V^{\prime}[F]_{\sigma}
$$

for all vector fields $V$ on $\mathcal{T}$ and all $\sigma \in \mathcal{T}$. We also get that $\partial \beta_{\sigma}(V)=0$, but in the non-compact case we cannot in general use this to get the vanishing of $\beta$. However, if we now define

$$
u(V)=\frac{1}{2 k+n}\left\{\frac{1}{2} \Delta_{G(V)}-\nabla_{G(V) d F}+2 k\left(V^{\prime}[F]+\beta(V)\right)\right\},
$$

then this $u$ solves (4) and hence gives us the existence of the Hitchin connection in the non-compact case as promised in the second remark in the introduction.

In the following sections we will be interested in the induced connection $\hat{\nabla}^{e}$ in the endomorphism bundle $\operatorname{End}\left(H^{(k)}\right)$. Suppose $\Phi$ is a section of $\operatorname{End}\left(H^{(k)}\right)$. Then for all sections $s$ of $H^{(k)}$ and all vector fields $V$ on $\mathcal{T}$, we have that

$$
\left(\hat{\nabla}_{V}^{e} \Phi\right)(s)=\hat{\nabla}_{V} \Phi(s)-\Phi\left(\hat{\nabla}_{V}(s)\right)
$$

Assume now that we have extended $\Phi$ to a section of $\operatorname{Hom}\left(\mathscr{H}^{(k)}, H^{(k)}\right)$ over $\mathcal{T}$. Then

$$
\hat{\nabla}_{V}^{e} \Phi=\hat{\nabla}_{V}^{e, t} \Phi+[\Phi, u(V)]
$$

where $\hat{\nabla}^{e, t}$ is the trivial connection in the trivial bundle End $\left(\mathscr{H}^{(k)}\right)$ over $\mathcal{T}$.

Lemma 2.12. There exists smooth one-forms $X_{r}, Z$ and functions $Y_{r}, r=1, \ldots, R$, on $\mathcal{T}$ with values in $C^{\infty}\left(M, T_{\sigma}\right)$ such that

$$
\frac{1}{2} \Delta_{G(V)}-\nabla_{G(V) d F}=\sum_{r=1}^{R} \nabla_{X_{r}(V)} \nabla_{Y_{r}}+\nabla_{Z(V)}
$$

for all vector fields $V$ on $\mathcal{T}$.

Proof. We fix a finite partition of unity $\left(U_{i}, \rho_{i}\right)$ of $M$, such that each $U_{i}$ is a contractible coordinate neighborhood $\left(U_{i}, x_{i}\right)$. Let $\tilde{\rho}_{i}$ be a smooth function with compact support in $U_{i}$ which is constant 1 on the support of $\rho_{i}$. Using the coordinate $x_{i}$ we get 
a trivialization of $T\left(U_{i}\right)$. By combining these with the projections onto the varying holomorphic tangent sub-bundle, we get a smoothly varying trivialization of these. Using this we see that we for each $i$ can find smooth one forms $X_{i}^{(j)}$ and $Y_{i}^{(j)}$ on $\mathcal{T}$ with values in $C^{\infty}\left(U_{i}, T\left(U_{i}\right) \otimes \mathbb{C}\right)$, which maps $T_{\sigma} \mathcal{T}$ to $C^{\infty}\left(M, T_{\sigma}\right)$ for all $\sigma \in \mathcal{T}$ and such that

$$
\left.G(V)\right|_{U_{i}}=2 \sum_{j} X_{i}^{(j)}(V) Y_{i}^{(j)}
$$

and hence

$$
G(V)=2 \sum_{i} \sum_{j} \rho_{i} X_{i}^{(j)}(V) \tilde{\rho}_{i} Y_{i}^{(j)} .
$$

Thus we conclude there exists smooth one-forms $X_{r}$ and functions $Y_{r}, r=1, \ldots, R$, on $\mathcal{T}$ with values in $C^{\infty}\left(M, T_{\sigma}\right)$ such that

$$
G(V)=2 \sum_{r=1}^{R} X_{r}(V) Y_{r}
$$

We now compute that

$$
\begin{aligned}
& \frac{1}{2} \Delta_{G(V)}-\nabla_{G(V) d F} \\
& \quad=\sum_{r=1}^{R} \nabla_{X_{r}(V)} \nabla_{Y_{r}}+\sum_{r=1}^{R} \operatorname{Tr}\left(\nabla\left(X_{r}(V)\right)\right) \nabla_{Y_{r}}-2 \sum_{r=1}^{R} X_{r}(V)(F) \nabla_{Y_{r}} .
\end{aligned}
$$

From this we see that

$$
Z(V)=\sum_{r=1}^{R} \operatorname{Tr}\left(\nabla\left(X_{r}(V)\right)\right) Y_{r}-2 \sum_{r=1}^{R} X_{r}(V)(F) Y_{r} .
$$

This gives us the expression

$$
u(V)=\frac{1}{2 k+n}\left(\sum_{r=1}^{R} \nabla_{X_{r}(V)} \nabla_{Y_{r}}+\nabla_{Z(V)}-n V^{\prime}[F]\right)+V^{\prime}[F] .
$$

All we need to use about $F: \mathcal{T} \rightarrow C^{\infty}(M)$ below is that it is a smooth function, such that $F_{\sigma}$ is real valued on $M$ for all $\sigma \in \mathcal{T}$.

Suppose $\Gamma$ is a group which acts by bundle automorphisms of $\mathscr{L}$ over $M$ preserving both the Hermitian structure and the connection in $\mathscr{L}$. Then there is an induced action of $\Gamma$ on $(M, \omega)$. We will further assume that $\Gamma$ acts on $\mathcal{T}$ and that $I$ is $\Gamma$-equivariant. In this case we immediately get the following invariance.

Lemma 2.13. The natural induced action of $\Gamma$ on $\mathscr{H}^{(k)}$ preserves the subbundle $H^{(k)}$ and the Hitchin connection. 


\section{Berezin-Toeplitz deformation quantization on compact Kähler manifolds}

For each $f \in C^{\infty}(M)$ we consider the prequantum operator, namely the differential operator $P_{f}^{(k)}: C^{\infty}\left(M, L^{k}\right) \rightarrow C^{\infty}\left(M, L^{k}\right)$ given by

$$
P_{f}^{(k)}=-\frac{1}{k} \nabla_{X_{f}}+i f .
$$

where $X_{f}$ is the Hamiltonian vector field associated to $f$.

These operators acts on $C^{\infty}\left(M, \mathscr{L}^{k}\right)$ and therefore also on the bundle $\mathscr{H}^{(k)}$, however, they do not preserve the subbundle $H^{(k)}$. In order to turn these operators into operators which act on $H^{(k)}$ we need to consider the Hilbert space structure.

Integrating the inner product of two sections against the volume form associated to the symplectic form gives the pre-Hilbert space structure on $C^{\infty}\left(M, \mathscr{L}^{k}\right)$

$$
\left\langle s_{1}, s_{2}\right\rangle=\frac{1}{m !} \int_{M}\left(s_{1}, s_{2}\right) \omega^{m} .
$$

We think of this as a pre-Hilbert space structure on the trivial bundle $\mathscr{H}^{(k)}$ which of course is compatible with the trivial connection in this bundle. This pre-Hilbert space structure induces a Hermitian structure $\langle\cdot, \cdot\rangle$ on the finite rank subbundle $H^{(k)}$ of $\mathscr{H}^{(k)}$. The Hermitian structure $\langle\cdot, \cdot\rangle$ on $H^{(k)}$ also induces the operator norm $\|\cdot\|$ on $\operatorname{End}\left(H^{(k)}\right)$.

Since $H_{\sigma}^{(k)}$ is a finite-dimensional subspace of $C^{\infty}\left(M, \mathscr{L}^{k}\right)=\mathscr{H}_{\sigma}^{(k)}$ and therefore closed, we have the orthogonal projection $\pi_{\sigma}^{(k)}: \mathscr{H}_{\sigma}^{(k)} \rightarrow H_{\sigma}^{(k)}$. Since $H^{(k)}$ is a smooth subbundle of $\mathscr{H}^{(k)}$ the projections $\pi_{\sigma}^{(k)}$ form a smooth map $\pi^{(k)}$ from $\mathcal{T}$ to the space of bounded operators on the $L_{2}$-completion of $C^{\infty}\left(M, \mathscr{L}^{k}\right)$. The easiest way to see this is to consider a local frame for $\left(s_{1}, \ldots s_{\operatorname{Rank}} H^{(k)}\right)$ of $H^{(k)}$. Let $h_{i j}=\left\langle s_{i}, s_{j}\right\rangle$. Let $h_{i j}^{-1}$ be the inverse matrix of $h_{i j}$. Then

$$
\pi_{\sigma}^{(k)}(s)=\sum_{i, j}\left\langle s,\left(s_{i}\right)_{\sigma}\right\rangle\left(h_{i j}^{-1}\right)_{\sigma}\left(s_{j}\right)_{\sigma} .
$$

This formula will be useful when we have to compute the derivative of $\pi^{(k)}$ along vector fields on $\mathcal{T}$.

From these projections we can construct the Toeplitz operators associated to any smooth function $f \in C^{\infty}(M), T_{f, \sigma}^{(k)}: \mathscr{H}_{\sigma}^{(k)} \rightarrow H_{\sigma}^{(k)}$, defined by

$$
T_{f, \sigma}^{(k)}(s)=\pi_{\sigma}^{(k)}(f s)
$$

for any element $s$ in $\mathscr{H}_{\sigma}^{(k)}$ and any point $\sigma \in \mathcal{T}$. We observe that the Toeplitz operators are smooth sections $T_{f}^{(k)}$ of the bundle $\operatorname{Hom}\left(\mathscr{H}^{(k)}, H^{(k)}\right)$ and restrict to smooth sections of $\operatorname{End}\left(H^{(k)}\right)$. 
Remark 3.1. Similarly for any pseudo-differential operator $A$ on $M$ with coefficients in $\mathscr{L}^{k}$ (which may even depend on $\sigma \in \mathcal{T}$ ), we can consider the associated Toeplitz operator $\pi^{(k)} A$ and think of it as a section of $\operatorname{Hom}\left(\mathscr{H}^{(k)}, H^{(k)}\right)$. However, whenever we consider asymptotic expansions of such or consider their operator norms, we implicitly restrict them to $H^{(k)}$ and consider them as section of $\operatorname{End}\left(H^{(k)}\right)$ or equivalently assume that they have been precomposed with $\pi^{(k)}$.

We recall by Tuynman's Theorem [49] that if we compose the prequantum operator associated to $f$ by the orthogonal projection, then it can be rewritten as a Toeplitz operator.

Theorem 3.2 (Tuynman). For any $f \in C^{\infty}(M)$ and any point $\sigma \in \mathcal{T}$ we have that

$$
\pi_{\sigma}^{(k)} \circ P_{f}^{(k)}=i T_{f-\frac{1}{2 k} \Delta_{\sigma} f, \sigma}^{(k)}
$$

as operators from $\mathscr{H}_{\sigma}^{(k)}$ to $H_{\sigma}^{(k)}$, where $\Delta_{\sigma}$ is the Laplacian on $\left(M_{\sigma}, \omega\right)$.

Tuynman's formula is of course equivalent to

$$
\pi_{\sigma}^{(k)} \nabla_{X_{f}}=T_{\frac{i}{2} \Delta_{\sigma}(f), \sigma}^{(k)} .
$$

This formula is really a corollary of a more general formula which we will need.

Suppose we have a smooth section $X \in C^{\infty}\left(M, T_{\sigma}\right)$ of the holomorphic tangent bundle of $M_{\sigma}$. We then claim that the operator $\pi^{(k)} \nabla_{X}$ is a zero-order Toeplitz operator. Suppose $s_{1} \in C^{\infty}\left(M, \mathscr{L}^{k}\right)$ and $s_{2} \in H^{0}\left(M_{\sigma}, \mathscr{L}^{k}\right)$, then we have that

$$
X\left(s_{1}, s_{2}\right)=\left(\nabla_{X} s_{1}, s_{2}\right) .
$$

Now, calculating the Lie derivative along $X$ of $\left(s_{1}, s_{2}\right) \omega^{m}$ and using the above, one obtains after integration that

$$
\left\langle\nabla_{X} s_{1}, s_{2}\right\rangle=-\left\langle\Lambda d\left(i_{X} \omega\right) s_{1}, s_{2}\right\rangle,
$$

where $\Lambda$ denotes contraction with $\omega$. Thus

$$
\pi^{(k)} \nabla_{X}=T_{f_{X}}^{(k)},
$$

as operators from $C^{\infty}\left(N, L^{k}\right)$ to $H^{0}\left(N, L^{k}\right)$, where $f_{X}=-\Lambda d\left(i_{X} \omega\right)$.

Tuynman's formula above now follows from

$$
\Lambda d\left(i_{\left(X_{f}\right)^{(1,0)}} \omega\right)=-\Lambda d\left(\bar{\partial}_{\sigma} f\right)=-\frac{i}{2} \Delta_{\sigma} f .
$$

Iterating (13), we find for all $X_{1}, X_{2} \in C^{\infty}\left(M, T_{\sigma}\right)$ that

$$
\pi^{(k)} \nabla_{X_{1}} \nabla_{X_{2}}=T_{f_{X_{2}} f_{X_{1}}-X_{2}\left(f_{X_{1}}\right)}^{(k)}
$$


again as operators from $C^{\infty}\left(M, \mathscr{L}^{k}\right)$ to $H^{0}\left(M_{\sigma}, \mathscr{L}^{k}\right)$.

For $X \in C^{\infty}\left(M, T_{\sigma}\right)$, the complex conjugate vector field $\bar{X} \in C^{\infty}\left(M, \bar{T}_{\sigma}\right)$ is a section of the antiholomorphic tangent bundle, and for $s_{1}, s_{2} \in C^{\infty}\left(M, \mathscr{L}^{k}\right)$, we have that

$$
\bar{X}\left(s_{1}, s_{2}\right)=\left(\nabla_{\bar{X}} s_{1}, s_{2}\right)+\left(s_{1}, \nabla_{X} s_{2}\right) .
$$

Computing the Lie derivative along $\bar{X}$ of $\left(s_{1}, s_{2}\right) \omega^{m}$ and integrating, we get that

$$
\left\langle\nabla_{\bar{X}} s_{1}, s_{2}\right\rangle+\left\langle\left(\nabla_{X}\right)^{*} s_{1}, s_{2}\right\rangle=-\left\langle\Lambda d\left(i_{\bar{X}} \omega\right) s_{1}, s_{2}\right\rangle
$$

Hence we see that

$$
\left(\nabla_{X}\right)^{*}=-\left(\nabla_{\bar{X}}-f_{\bar{X}}\right)
$$

as operators on $C^{\infty}\left(M, \mathscr{L}^{k}\right)$. In particular, we see that

$$
\pi^{(k)}\left(\nabla_{X}\right)^{*} \pi^{(k)}=-\left.T_{f_{\bar{X}}}^{(k)}\right|_{H^{0}\left(M_{\sigma}, \mathscr{L}^{k}\right)}: H^{0}\left(M_{\sigma}, \mathscr{L}^{k}\right) \rightarrow H^{0}\left(M_{\sigma}, \mathscr{L}^{k}\right) .
$$

For two smooth sections $X_{1}, X_{2}$ of the holomorphic tangent bundle $T_{\sigma}$ and a smooth function $h \in C^{\infty}(M)$, we deduce from the formula for $\left(\nabla_{X}\right)^{*}$ that

$$
\begin{gathered}
\pi^{(k)}\left(\nabla_{X_{1}}\right)^{*}\left(\nabla_{X_{2}}\right)^{*} h \pi^{(k)}=\pi^{(k)} \\
\bar{X}_{1} \bar{X}_{2}(h) \pi^{(k)}-\pi^{(k)} f_{\bar{X}_{1}} \bar{X}_{2}(h) \pi \\
-\pi f_{\bar{X}_{2}} \bar{X}_{1}(h) \pi^{(k)}-\pi^{(k)} \bar{X}_{1}\left(f_{\bar{X}_{2}}\right) h \pi \\
+\pi f_{\bar{X}_{1}} f_{\bar{X}_{2}} h \pi^{(k)}
\end{gathered}
$$

as operators on $H^{0}\left(M_{\sigma}, \mathscr{L}^{k}\right)$.

The product of two Toeplitz operators associated to two smooth functions will in general not be the Toeplitz operator associated to a smooth function again for all levels $k$, but there is an asymptotic expansion of the product in terms of such Toeplitz operators on a compact Kähler manifold by the results of Schlichenmaier [44].

Theorem 3.3 (Schlichenmaier). For any pair of smooth functions $f_{1}, f_{2} \in C^{\infty}(M)$, we have an asymptotic expansion

$$
T_{f_{1}, \sigma}^{(k)} T_{f_{2}, \sigma}^{(k)} \sim \sum_{l=0}^{\infty} T_{c_{\sigma}^{(l)}\left(f_{1}, f_{2}\right), \sigma}^{k^{-l}}
$$

where $c_{\sigma}^{(l)}\left(f_{1}, f_{2}\right) \in C^{\infty}(M)$ are uniquely determined since $\sim$ means the following: for all $L \in \mathbb{Z}_{+}$we have that

$$
\left\|T_{f_{1}, \sigma}^{(k)} T_{f_{2}, \sigma}^{(k)}-\sum_{l=0}^{L} T_{c_{\sigma}^{(l)}\left(f_{1}, f_{2}\right), \sigma}^{(k)} k^{-l}\right\|=O\left(k^{-(L+1)}\right)
$$

uniformly over compact subsets of $\mathcal{T}$. Moreover, $c_{\sigma}^{(0)}\left(f_{1}, f_{2}\right)=f_{1} f_{2}$. 
Remark 3.4. It will be useful for us to define new coefficients $\tilde{c}_{\sigma}^{(l)}(f, g) \in C^{\infty}(M)$ which correspond to the expansion of the product in $1 /(2 k+n)$ (where $n$ is some fixed integer):

$$
T_{f_{1}, \sigma}^{(k)} T_{f_{2}, \sigma}^{(k)} \sim \sum_{l=0}^{\infty} T_{\tilde{c}_{\sigma}^{(l)}\left(f_{1}, f_{2}\right), \sigma}^{(k)}(2 k+n)^{-l} .
$$

Theorem 3.3 is proved in [44], where it is also proved that the formal generating series for the $c_{\sigma}^{(l)}\left(f_{1}, f_{2}\right)$ 's gives a formal deformation quantization ${ }^{3}$ of symplectic manifold $(M, \omega)$.

We recall the definition of a formal deformation quantization. Introduce the space of formal functions $C_{h}^{\infty}(M)=C^{\infty}(M) \llbracket h \rrbracket$ as the space of formal power series in the variable $h$ with coefficients in $C^{\infty}(M)$. Let $\mathbb{C}_{h}=\mathbb{C} \llbracket h \rrbracket$.

Definition 3.5. A deformation quantization of $(M, \omega)$ is an associative product $*$ on $C_{h}^{\infty}(M)$ which respects the $\mathbb{C}_{h}$-module structure. It is determined by a sequence of bilinear operators

$$
c^{(l)}: C^{\infty}(M) \otimes C^{\infty}(M) \longrightarrow C^{\infty}(M)
$$

defined through

$$
f * g=\sum_{l=0}^{\infty} c^{(l)}(f, g) h^{l},
$$

where $f, g \in C^{\infty}(M)$. The deformation quantization is said to be differential, if the operators $c^{(l)}$ are bidifferential operators. Considering the symplectic action of $\Gamma$ on $(M, \omega)$, we say that a $*$-product $*$ is $\Gamma$-invariant if

$$
\gamma^{*}(f * g)=\gamma^{*}(f) * \gamma^{*}(g)
$$

for all $f, g \in C^{\infty}(M)$ and all $\gamma \in \Gamma$.

Theorem 3.6 (Karabegov and Schlichenmaier). The product $\star_{\sigma}^{B T}$ given by

$$
f \star_{\sigma}^{B T} g=\sum_{l=0}^{\infty}(-1)^{l} c_{\sigma}^{(l)}(f, g) h^{l},
$$

where $f, g \in C^{\infty}(M)$ and $c_{\sigma}^{(l)}(f, g)$ are determined by Theorem 3.3, is a differential deformation quantization of $(M, \omega)$.

Definition 3.7. The Berezin-Toeplitz deformation quantization of the compact Kähler manifold $\left(M_{\sigma}, \omega\right)$ is the product $\star_{\sigma}^{\mathrm{BT}}$.

\footnotetext{
${ }^{3}$ We have the opposite sign-convention on the curvature, which means our $c_{l}$ are $(-1)^{l} c_{l}$ in [44].
} 
Remark 3.8. Let $\Gamma_{\sigma}$ be the $\sigma$-stabilizer subgroup of $\Gamma$. For any element $\gamma \in \Gamma_{\sigma}$, we have that

$$
\gamma^{*}\left(T_{f, \sigma}^{(k)}\right)=T_{\gamma^{*} f, \sigma}^{(k)}
$$

This implies the invariance of $\star_{\sigma}^{\mathrm{BT}}$ under the $\sigma$-stabilizer $\Gamma_{\sigma}$.

Remark 3.9. We define a new $*$-product by

$$
f \tilde{\star}_{\sigma}^{\mathrm{BT}} g=\sum_{l=0}^{\infty}(-1)^{l} \tilde{c}_{\sigma}^{(l)}(f, g) h^{l} .
$$

Then

$$
f \tilde{\star}_{\sigma}^{\mathrm{BT}} g=\left(\left(f \circ \varphi^{-1}\right) \star_{\sigma}^{\mathrm{BT}}\left(g \circ \varphi^{-1}\right)\right) \circ \varphi
$$

for all $f, g \in C_{h}^{\infty}(M)$, where $\varphi(h)=\frac{h}{2+n h}$.

In [32], this Berezin-Toeplitz deformation quantization is identified in terms of Karabegov's classification of $*$-products with separation of variables on Kähler manifolds. Adopting the convention where the roles of holomorphic and anti-holomorphic are interchanged in the condition for a star product to be with separation of variables from [32], one of the main result of that paper reads

Theorem 3.10 (Karabegov and Schlichenmaier). The Karabegov form $\tilde{\omega}_{\sigma}$ of the Berezin-Toeplitz *-product $\star_{\sigma}^{B T}$ is

$$
\tilde{\omega}_{\sigma}=\frac{1}{h} \omega+\operatorname{Ric}_{\sigma}
$$

We will also need the following theorem due to Bordemann, Meinrenken and Schlichenmaier (see [22]).

Theorem 3.11 (Bordemann, Meinrenken, and Schlichenmaier). For any $f \in C^{\infty}(M)$ we have that

$$
\lim _{k \rightarrow \infty}\left\|T_{f, \sigma}^{(k)}\right\|=\sup _{x \in M}|f(x)|
$$

Since the association of the sequence of Toeplitz operators $T_{f, \sigma}^{(k)}, k \in \mathbb{Z}_{+}$is linear in $f$, we see from this theorem, that this association is faithful. 


\section{The formal Hitchin connection}

We assume the conditions on $(M, \omega)$ and $I$ of Theorem 2.4, thus providing us with a Hitchin connection $\hat{\nabla}$ in $H^{(k)}$ over $\mathcal{\mathcal { T }}$ and the associated connection $\hat{\nabla}^{e}$ in $\operatorname{End}\left(H^{(k)}\right)$.

Before proving Theorem 1.8, we need to establish some basic properties.

First, we need a useful formula for the derivative of the orthogonal projection $\pi^{(k)}$ along a curve $\sigma_{t}$ in $\mathcal{T}$. To this end, consider a basis of covariant constant sections $\left(s_{i}\right)_{t}$, $i=1, \ldots$, Rank $H^{(k)}$, of $H^{(k)}$ over a curve $\sigma_{t}$ in $\mathcal{T}$ :

$$
\left(s_{i}\right)_{t}^{\prime}=u\left(\sigma_{t}^{\prime}\right)\left(\left(s_{i}\right)_{t}\right), \quad i=1, \ldots, \operatorname{Rank} H^{(k)} .
$$

Recall formula (12) for the projection $\pi^{(k)}: \mathscr{H}_{\sigma}^{(k)} \rightarrow H_{\sigma}^{(k)}$ and compute the derivative along $\sigma_{t}$ : for any fixed $s \in C^{\infty}\left(M, L^{k}\right)$, we have that

$$
\begin{aligned}
\left(\pi_{\sigma_{t}}^{(k)}\right)^{\prime}(s)=\sum_{i, j}\left\langle s,\left(s_{i}\right)_{t}^{\prime}\right\rangle\left(h_{i j}^{-1}\right)_{t}\left(s_{j}\right)_{t}+\sum_{i, j}\langle & \left.s,\left(s_{i}\right)_{t}\right\rangle\left(h_{i j}^{-1}\right)_{t}^{\prime}\left(s_{j}\right)_{t} \\
& +\sum_{i, j}\left\langle s,\left(s_{i}\right)_{t}\right\rangle\left(h_{i j}^{-1}\right)_{t}\left(s_{j}\right)_{t}^{\prime} .
\end{aligned}
$$

An easy computation gives that

$$
\left(h_{i j}^{-1}\right)_{t}^{\prime}=-\sum_{l, r}\left(h_{i l}^{-1}\right)_{t}\left(\left\langle\left(s_{l}\right)_{t}^{\prime},\left(s_{r}\right)_{t}\right\rangle+\left\langle\left(s_{l}\right)_{t},\left(s_{r}\right)_{t}^{\prime}\right\rangle\right)\left(h_{r j}^{-1}\right)_{t},
$$

so

$$
\begin{aligned}
\pi_{\sigma_{t}}^{(k)}\left(\pi_{\sigma_{t}}^{(k)}\right)^{\prime}(s)= & \sum_{i, j}\left\langle u_{G\left(\sigma_{t}^{\prime}\right)}^{*} s,\left(s_{i}\right)_{t}\right\rangle\left(h_{i j}^{-1}\right)_{t}\left(s_{j}\right)_{t} \\
& \quad-\sum_{i, l, m, j}\left\langle s,\left(s_{i}\right)_{t}\right\rangle\left(h_{i l}^{-1}\right)_{t}\left\langle\left(s_{l}\right)_{t},\left(s_{m}\right)_{t}^{\prime}\right\rangle\left(h_{m j}^{-1}\right)_{t}\left(s_{j}\right)_{t} \\
= & \pi_{\sigma_{t}}^{(k)} u\left(\sigma_{t}^{\prime}\right)^{*}(s)-\sum_{m, j}\left\langle\pi_{\sigma_{t}}^{(k)} s, u\left(\sigma_{t}^{\prime}\right)\left(\left(s_{i}\right)_{t}\right)\right\rangle\left(h_{m j}^{-1}\right)_{t}\left(s_{j}\right)_{t} \\
= & \pi_{\sigma_{t}}^{(k)} u\left(\sigma_{t}^{\prime}\right)^{*}(s)-\pi_{\sigma_{t}}^{(k)} u\left(\sigma_{t}^{\prime}\right)^{*} \pi_{\sigma_{t}}^{(k)}(s) .
\end{aligned}
$$

Hence we conclude that

Lemma 4.1. For any smooth vector field $V$ on $\mathcal{T}$, we have that

$$
\pi^{(k)} V\left[\pi^{(k)}\right]=\pi^{(k)} u(V)^{*}-\pi^{(k)} u(V)^{*} \pi^{(k)} .
$$

Let us now apply this formula for the derivative. 
Proof of Theorem 1.8. Using formula (9) we see that

$$
\hat{\nabla}_{V}^{e} T_{f}^{(k)}=V\left[T_{f}^{(k)}\right]+\left[T_{f}^{(k)}, u(V)\right]
$$

and so we compute

$$
\begin{aligned}
\pi^{(k)} \hat{\nabla}_{V}^{e} T_{f}^{(k)} \pi^{(k)}= & \pi^{(k)} V[f] \pi^{(k)}+\pi^{(k)} V\left[\pi^{(k)}\right] f \pi^{(k)} \\
& -\pi^{(k)} u(V) \pi^{(k)} f \pi^{(k)}+\pi^{(k)} f u(V) \pi^{(k)} \\
= & \pi^{(k)} V[f] \pi^{(k)}+\pi^{(k)} V^{\prime \prime}[F] f \pi^{(k)}-\pi^{(k)} V^{\prime \prime}[F] \pi^{(k)} f \pi^{(k)} \\
+ & \pi^{(k)} V^{\prime}[F] f \pi^{(k)}-\pi^{(k)} V^{\prime}[F] \pi^{(k)} f \pi^{(k)} \\
& +\frac{1}{2 k+n}\left(\pi^{(k)} o(V)^{*} f \pi^{(k)}-\pi^{(k)} o(V)^{*} \pi^{(k)} f \pi^{(k)}\right. \\
& \left.+\pi^{(k)} f o(V) \pi^{(k)}-\pi^{(k)} o(V) \pi^{(k)} f \pi^{(k)}\right) .
\end{aligned}
$$

Now, by combining (11) with (13) to (16), we get a one-form $E$ on $\mathcal{T}$ with values in $\mathscr{D}(M)$ such that

$$
T_{E(V) f}^{(k)}=\pi^{(k)} o(V)^{*} f \pi^{(k)}+\pi^{(k)} f o(V) \pi^{(k)} .
$$

Let $H$ be the one-form on $\mathcal{T}$ with values in $C^{\infty}(M)$ such that $H(V)=E(V)(1)$. Then we get the formula

$$
\begin{aligned}
\pi^{(k)} & \hat{\nabla}_{V}^{e} T_{f}^{(k)} \pi^{(k)} \\
& =T_{V[f]}^{(k)}+\left(T_{V[F] f}^{(k)}-T_{V[F]}^{(k)} T_{f}^{(k)}\right)+\frac{1}{2 k+n}\left(T_{E(V)(f)}^{(k)}-T_{H(V)}^{(k)} T_{f}^{(k)}\right) .
\end{aligned}
$$

From this we obtain, using Theorem 3.3, the wanted estimate by letting

$$
\tilde{D}(V)(f)=V[F] f-V[F] \tilde{\star}^{\mathrm{BT}} f+h\left(E(V)(f)-H(V) \tilde{\star}^{\mathrm{BT}} f\right)
$$

which is clearly divisible by $h$. The uniqueness of $\widetilde{D}$ follows from Theorem 3.11 .

Lemma 4.2. If $A$ is a smooth family of Toeplitz operators of order $d$, then $\hat{\nabla}_{V}^{e} A$ is also a smooth family of Toeplitz operators of order $d$ and

$$
\sigma_{d}\left(\hat{\nabla}_{V}^{e} A\right)=V\left[\sigma_{d}(A)\right]
$$

for any vector field $V$ on $\mathcal{T}$.

Proof. Since $\hat{\nabla}_{V}^{e} A$ is again a smooth section of $\operatorname{End}\left(H^{(k)}\right)$ over $\mathcal{T}$, we have that

$$
\hat{\nabla}_{V}^{e} A=\pi \hat{\nabla}_{V}^{e} A
$$

Now we can simply apply (17) to obtain the desired conclusion. 
Proposition 4.3. Suppose $f, g: \mathcal{T} \rightarrow C^{\infty}(M)$ are smooth functions. Then $T_{f}^{(k)} T_{g}^{(k)}$ is a smooth family of Toeplitz operators over $\mathcal{T}$ and for any vector field $V$ on $\mathcal{T}$ we have that

$$
\hat{\nabla}_{V}^{e}\left(T_{f}^{(k)} T_{g}^{(k)}\right) \sim \hat{\nabla}_{V}^{e}\left(T_{f \star^{B T} g}^{(k)}\right),
$$

i.e. for all $L \in \mathbb{Z}_{+}$we have that

$$
\left\|\hat{\nabla}_{V}^{e}\left(T_{f}^{(k)} T_{g}^{(k)}\right)-\sum_{l=0}^{L} \hat{\nabla}_{V}^{e}\left(T_{c_{l}(f, g)}^{(k)}\right)\right\|=O\left(k^{-(L+1)}\right) .
$$

Proof. We use the notation of [44] and [45] and the setup of generalized Toeplitz operators described there. In this set-up one assigns to the prequantum line bundle the unit circle bundle of the dual bundle. The above Toeplitz operators $T_{f}^{(k)}$ are the components (with respect to the circle action) of a zero order generalized Toeplitz operator $T_{f}$. The generator of the circle action $D_{\varphi}$ is a generalized Toeplitz operator of order one. We let

$$
A_{L}=D_{\varphi}^{L} T_{f} T_{g}-\sum_{l=0}^{L} D_{\varphi}^{L-l} T_{c_{l}(f, g)} .
$$

Suppose $V$ be a vector field on $\mathcal{T}$. We will now establish by induction that $\hat{\nabla}^{e} A_{L}$ is a zero order Toeplitz operator and

$$
\sigma_{0}\left(\hat{\nabla}_{V}^{e} A_{L}\right)=V\left[c_{L}(f, g)\right] .
$$

Note that Lemma 4.2 implies this claim for $A_{0}=T_{f} T_{g}$, since we have just argued this operator is smooth. Assume we have established this claim for $\hat{\nabla}^{e} A_{L-1}$. Since

$$
A_{L}=D_{\varphi} A_{L-1}-D_{\varphi} T_{c_{L-1}(f, g)},
$$

we see that $A_{L}$ is a smooth family of Toeplitz operators parameterized by $\mathcal{T}$ and

$$
\hat{\nabla}_{V}^{e} A_{L}=D_{\varphi} \hat{\nabla}_{V}^{e} A_{L-1}-D_{\varphi} \hat{\nabla}_{V}^{e} T_{c_{L-1}(f, g)} .
$$

We see this is at most a first order operator by induction, but we have

$$
\sigma_{1}\left(\hat{\nabla}_{V}^{e} A_{L}\right)=t\left(\sigma_{0}\left(\hat{\nabla}_{V}^{e} A_{L-1}\right)-V\left[c_{L-1}(f, g)\right]\right)=0
$$

by the previous lemma, so it is at most a 0 -order operator. Applying the previous lemma again we see that

$$
\sigma_{0}\left(\hat{\nabla}_{V}^{e} A_{L}\right)=V\left[\sigma_{0}\left(A_{L}\right)\right]=V\left[c_{L}(f, g)\right] .
$$

This completes the inductive step.

The estimates of the theorem now follow since by induction $\hat{\nabla}_{V}^{e} A_{L}$ is a zero order Toeplitz operator for all $L \in \mathbb{Z}_{+}$. 
Lemma 4.4. The formal operator $D_{V}$ is a derivation for $\star_{\sigma}^{B T}$ for each $\sigma \in \mathcal{T}$, i.e.

$$
D_{V}\left(f \star^{B T} g\right)=D_{V}(f) \star^{B T} g+f \star^{B T} D_{V}(g)
$$

for all $f, g \in C^{\infty}(M)$.

Proof. By definition of $\star_{\sigma}^{\mathrm{BT}}$ we have for all $\sigma \in \mathcal{T}$ that

$$
T_{f, \sigma}^{(k)} T_{g, \sigma}^{(k)} \sim T_{f \star{ }_{\sigma}^{\mathrm{BT}} g, \sigma}^{(k)}
$$

Let $V$ be a vector field on $\mathcal{T}$. By Proposition 4.3 we have that

$$
\hat{\nabla}_{V}^{e}\left(T_{f}^{(k)} T_{g}^{(k)}\right) \sim \hat{\nabla}_{V}^{e}\left(T_{f \star \mathrm{BT} g}^{(k)}\right) .
$$

Considering the left hand side, we see that

$$
\hat{\nabla}_{V}^{e}\left(T_{f}^{(k)} T_{g}^{(k)}\right)=\hat{\nabla}_{V}^{e}\left(T_{f}^{(k)}\right) T_{g}^{(k)}+T_{f}^{(k)} \hat{\nabla}_{V}^{e}\left(T_{g}^{(k)}\right)
$$

Now apply Theorem 1.8 to get the wanted conclusion.

Proposition 4.5. For two vector fields $V_{1}, V_{2}$ on $\mathcal{T}$, we have the formula

$$
\left(\left[\hat{\nabla}_{V_{1}}^{e}, \hat{\nabla}_{V_{2}}^{e}\right]-\hat{\nabla}_{\left[V_{1}, V_{2}\right]}^{e}\right)\left(T_{f}^{(k)}\right) \sim T_{\left(\left[D_{V_{1}}, D_{V_{2}}\right]-D_{\left[V_{1}, V_{2}\right]}^{(k)}\right)(f)(1 /(2 k+n))}^{(k)}
$$

From this proposition we conclude in particular that flatness of $\hat{\nabla}^{e}$ implies flatness of $D$.

Proof. By (18) we see that

$$
\begin{aligned}
\hat{\nabla}_{V_{1}}^{e}\left(\hat{\nabla}_{V_{2}}^{e}\left(T_{f}^{(k)}\right)\right)= & \hat{\nabla}_{V_{1}}^{e}\left(T_{V_{2}[f]}^{(k)}\right)+\hat{\nabla}_{V_{1}}^{e}\left(T_{V_{2}^{\prime}[F] f}^{(k)}\right)-\hat{\nabla}_{V_{1}}^{e}\left(T_{V_{2}^{\prime}[F]}^{(k)} T_{f}^{(k)}\right) \\
& +\frac{1}{2 k+n}\left(\hat{\nabla}_{V_{1}}^{e}\left(T_{E\left(V_{2}\right)(f)}^{(k)}\right)-\hat{\nabla}_{V_{1}}^{e}\left(T_{H\left(V_{2}\right)}^{(k)} T_{f}^{(k)}\right)\right)
\end{aligned}
$$

But now by applying first Proposition 4.3 and then Theorem 1.8 to this expression, we see that

$$
\hat{\nabla}_{V_{1}}^{e}\left(\hat{\nabla}_{V_{2}}^{e}\left(T_{f}^{(k)}\right)\right) \sim T_{D_{V_{1}} D_{V_{2}} f}^{(k)}
$$

The proposition then follows from this and Theorem 1.8. 


\section{Formal trivializations and symmetry-invariant $*$-products}

Recalling Definition 1.12, it is clear that a formal Hitchin connection $D$ will not have a formal trivialization even locally on $\mathcal{T}$, if it is not flat. However, if $D$ is flat, which is implied if $\hat{\nabla}$ is projectively flat by Proposition 4.5 , then we have the following result.

Proposition 5.1. Assume that $D$ is flat and that $\widetilde{D}=0 \bmod h$. Then locally around any point in $\mathcal{T}$ there exists a formal trivialization. If $H^{1}(\mathcal{T}, \mathbb{R})=0$ then there exists a formal trivialization defined globally on $\mathcal{T}$. If further $H_{\Gamma}^{1}(\mathcal{T}, \mathscr{D}(M))=0$ then we can construct $P$ such that it is $\Gamma$-equivariant.

In this Proposition $H_{\Gamma}^{1}(\mathcal{T}, \mathscr{D}(M))$ simply refers to the $\Gamma$-equivariant first de Rham cohomology of $\mathcal{T}$ with coefficients in the real $\Gamma$-vector space $\mathscr{D}(M)$.

Proof. We write the formal trivialization we seek as

$$
P=\sum_{l=0}^{\infty} P_{l} h^{l}
$$

where $P_{l}: \mathcal{T} \rightarrow \mathscr{D}(M)$. We need to solve

$$
D_{V} P=\sum_{l=0}^{\infty} V\left[P_{l}\right] h^{l}+\sum_{l=0}^{\infty} \sum_{r=1}^{l} \widetilde{D}^{(r)}(V) P_{l-r} h^{l} .
$$

Hence we need that

$$
V\left[P_{l}\right]=\sum_{r=1}^{l} \widetilde{D}^{(r)}(V) P_{l-r} .
$$

Now $P_{0}=$ Id solves this equation for $l=0$. Assume that we have solved (locally, globally on $\mathcal{T}$ respectively $\Gamma$-equivariantly on $\mathcal{T}$ ) this equation for $P_{r}$, for $r<l$. Then let $\alpha_{l} \in \Omega^{1}(\mathcal{T}, \mathscr{D}(M))$ be given by

$$
\alpha_{l}(V)=\sum_{r=1}^{l} \widetilde{D}^{(r)}(V) P_{l-r} .
$$

We observe that $\alpha_{l}$ is $\Gamma$-invariant. A short computation shows that the flatness of $D$ implies that $\alpha_{l}$ is closed on $\mathcal{T}$. Hence we can (locally, globally on $\mathcal{T}$ respectively $\Gamma$-equivariantly on $\mathcal{T}$ ) solve (21) for $P_{l}$.

Now suppose we have a formal trivialization $P$ of the formal Hitchin connection $D$ determined by (2). Then $P$ is constant $\bmod h$ and we may and will assume that

$$
P=\mathrm{Id} \bmod h .
$$


We can then define a new smooth family of star products, parametrized by $\mathcal{T}$, by

$$
f \star_{\sigma} g=P_{\sigma}^{-1}\left(P_{\sigma}(f) \star_{\sigma}^{\mathrm{BT}} P_{\sigma}(g)\right)
$$

for all $f, g \in C^{\infty}(M)$ and all $\sigma \in \mathcal{T}$.

Proposition 5.2. The star-products $\star_{\sigma}$ are independent of $\sigma \in \mathcal{T}$.

Proof. Let $f, g \in C^{\infty}(M)$. Since $D_{V}$ is a derivation for $\star_{\sigma}^{\mathrm{BT}}$ for any $\sigma \in \mathcal{T}$, we have that

$$
D_{V}\left(P_{\sigma}(f) \star_{\sigma}^{\mathrm{BT}} P_{\sigma}(g)\right)=0 .
$$

However we have

$$
\begin{array}{r}
D_{V}\left(P_{\sigma}(f) \star_{\sigma}^{\mathrm{BT}} P_{\sigma}(g)\right)=\tilde{D}(V)\left(P_{\sigma}(f) \star_{\sigma}^{\mathrm{BT}} P_{\sigma}(g)\right)+V\left[P_{\sigma}\right](f) \star_{\sigma}^{\mathrm{BT}} P_{\sigma}(g) \\
+P_{\sigma}(f) \star_{\sigma}^{\mathrm{BT}} V\left[P_{\sigma}\right](g)+P_{\sigma}(f) V\left[\star_{\sigma}^{\mathrm{BT}}\right] P_{\sigma}(g),
\end{array}
$$

which we compare with

$$
\begin{aligned}
V\left[f \star_{\sigma} g\right]=V & {\left[P_{\sigma}^{-1}\right]\left(P_{\sigma}(f) \star_{\sigma}^{\mathrm{BT}} P_{\sigma}(g)\right)+P_{\sigma}^{-1}\left(V\left[P_{\sigma}\right](f) \star_{\sigma}^{\mathrm{BT}} P_{\sigma}(g)\right) } \\
& +P_{\sigma}^{-1}\left(P_{\sigma}(f) \star_{\sigma}^{\mathrm{BT}} V\left[P_{\sigma}\right](g)\right)+P_{\sigma}^{-1}\left(P_{\sigma}(f) V\left[\star_{\sigma}^{\mathrm{BT}}\right] P_{\sigma}(g)\right)
\end{aligned}
$$

and conclude, since $P V\left[P^{-1}\right]=\widetilde{D}(V)$, that

$$
V\left[f \star_{\sigma} g\right]=0 .
$$

From the above we conclude Theorem 1.13.

Now, let us analyze equivalences between symmetry invariant $*$-products. Suppose we have two differential $\Gamma$-invariant $*$-products $*$ and $*^{\prime}$, which are equivalent under some equivalence

$$
T=\mathrm{Id}+\sum_{j=1}^{\infty} h^{j} T_{j},
$$

where $T_{j}: C^{\infty}(M) \rightarrow C^{\infty}(M)$ is a linear map for each $j \in \mathbb{N}$, such that

$$
T(f * g)=T(f) *^{\prime} T(g) .
$$

By Theorem 2.22 in [30] it follows that $T_{j}$ is a differential operator for all $j \in \mathbb{N}$.

Proposition 5.3. If the first discrete cohomology of $\Gamma$ with coefficient in the $\Gamma$-module $C_{0}^{\infty}(M), H^{1}\left(\Gamma, C_{0}^{\infty}(M)\right)$ and first de Rham cohomology of $M$ with real coefficients, $H^{1}(M, \mathbb{R})$, both vanish, then we can find a $\Gamma$-invariant equivalence between $*$ and $*^{\prime}$.

Please see our joint work with Villemoes [12], [13], and [14] where we prove the vanishing of the first cohomology of the mapping class group with coefficients in modules of functions (of various kinds) on moduli spaces. This is also related to our application in Section 6 below. 
Proof. We consider the given equivalence $T$ and by a short computation we get that

$$
T^{-1} \gamma^{*}(T)(f * g)=T^{-1} \gamma^{*}(T)(f) * T^{-1} \gamma^{*}(T)(g),
$$

hence $T^{-1} \gamma^{*}(T)$ is an automorphism of $*$. Since $H^{1}(M, \mathbb{R})=0$ we get by Proposition 3.3 in [30] that there exists $a_{\gamma} \in C_{h}^{\infty}(M)$ for each $\gamma \in \Gamma$ such that

$$
T^{-1} \gamma^{*}(T)=\exp \left(\operatorname{ad}_{*} a_{\gamma}\right) \text {. }
$$

We observe for $u, f \in C^{\infty}(M)$ that

$$
\operatorname{ad}_{*}(u)(f)=\{u, f\} h+O\left(h^{2}\right),
$$

and that $\operatorname{ad}_{*}(c)=0$ for all $c \in \mathbb{C}_{h}$. If we have that $a_{\gamma}=\sum_{j=0}^{\infty} a_{\gamma}^{(j)} h^{j}$, then we may assume that $a_{\gamma}^{(j)} \in C_{0}^{\infty}(M)$. Furthermore $a_{\gamma}$ is then uniquely determined by (22). Let us now assume that

$$
a_{\gamma}^{(i)}=0
$$

for all $\gamma \in \Gamma$ and $i=0, \ldots j-1$. We will then show that we can modify $T_{j}$ to obtain a new equivalence which through (22) produces a new $a_{\gamma}$, which vanishes modulo $h^{j+1}$.

First we see that

$$
\begin{aligned}
\exp \left(\operatorname{ad}_{*} a_{\gamma_{1} \gamma_{2}}\right) & =\exp \left(\operatorname{ad}_{*} a_{\gamma_{1}}\right) \exp \left(\operatorname{ad}_{*} \gamma_{1}^{*}\left(a_{\gamma_{2}}\right)\right) \\
& =\exp \left(\operatorname{ad}_{*}\left(a_{\gamma_{1}} \circ_{*} \gamma_{1}^{*}\left(a_{\gamma_{2}}\right)\right)\right)
\end{aligned}
$$

by Lemma 4.1 in [30], where $\circ_{*}$ is the Campbell-Baker-Hausdorff composition

$$
a \circ b=a+\int_{0}^{1} \psi\left(\exp \left(\operatorname{ad}_{*} a\right) \circ \exp \left(t \operatorname{ad}_{*} b\right)\right) b d t,
$$

where

$$
\psi(z)=\frac{z \log (z)}{z-1}
$$

One has for $a, b \in C^{\infty}(M)$ that

$$
a \circ_{*} b=a+b+O\left(h^{1}\right) .
$$

From the above we may conclude that

$$
a_{\gamma_{1} \gamma_{2}}^{(j)}=a_{\gamma_{1}}^{(j)}+\gamma_{1}^{*}\left(a_{\gamma_{2}}^{(j)}\right)
$$

Hence we see that

$$
\left(a_{\gamma}^{(j)}\right) \in Z^{1}\left(\Gamma, C_{0}^{\infty}(M)\right) .
$$

But by assumption $H^{1}\left(\Gamma, C_{0}^{\infty}(M)\right)=0$, so this means that there exists $a^{(j)} \in$ $C_{0}^{\infty}(M)$ such that

$$
a_{\gamma}^{(j)}=\gamma^{*}\left(a^{(j)}\right)-a^{(j)} .
$$


Now replace $T_{j}$ by $T_{j} \exp \left(\operatorname{ad}_{*}\left(a^{(j)} h^{j}\right)\right)$ and obtain a new equivalence which produces a new $a_{\gamma}$ with the required vanishing. By induction we have the conclusion of the proposition.

Remark 5.4. From this we conclude that if the commutant of $\Gamma$ in $D(M)$ is trivial, i.e. it contains only scalar multiples of the identity, then a $\Gamma$ invariant differential *-product on $M$ is unique.

\section{Applications to moduli space of flat connections}

Let $\Sigma$ be a compact oriented surface possibly with boundary. Let $\mathcal{M}$ be the moduli space of flat $\mathrm{SU}(n)$-connections on $\Sigma$

$$
\mathcal{M}=\operatorname{Hom}\left(\pi_{1}(\Sigma), \operatorname{SU}(n)\right) / \operatorname{SU}(n) .
$$

There is a natural Poisson structure on $\mathcal{M}$; see [27] and [28]. The symplectic leaves are specified by fixing the conjugacy-class of the holonomy around each component of the boundary of $\Sigma$. Let $(M, \omega)$ be a smooth symplectic leaf of $\mathcal{M}$. Pick a prequantum line bundle on $(M, \omega)$. Teichmüller space of $\Sigma$ parametrizes a complex family of complex structures on each $(M, \omega)$. Then the assumptions of Theorem 1.3 are satisfied, since Hitchin's proof from [31] generalizes word for word to this situation and shows that this family of complex structures is rigid. We get the existence of the Hitchin connection given by (1).

As a corollary of Lemma 2.8 (which gives an explicit expression for the function Hitchin calls $f_{G}$ in his paper [31]), we get that Hitchin's connection agrees with the connection constructed by Axelrod, Della Pietra and Witten in [17], as is stated in the introduction in Theorem 1.4.

Now the techniques used in [31] to show that the Hitchin connection is projectively flat apply in our situation, hence we conclude that the induced connection in the endomorphism bundle $\hat{\nabla}^{e}$ is flat. Since Teichmüller space is contractible, we can apply Theorem 1.10 to obtain Theorem 1.14 .

\section{References}

[1] J. E. Andersen, Deformation quantization and geometric quantization of abelian moduli spaces. Comm. Math. Phys. 255 (2005), 727-745. MR 2135450 Zbl 1079.53136

[2] J. E. Andersen, Asymptotic faithfulness of the quantum SU(n) representations of the mapping class groups. Ann. of Math. (2) 163 (2006), 347-368. MR 2195137 Zbl 1157.53049 annals.math.princeton.edu/2006/163-1/p07

[3] J. E. Andersen, The Nielsen-Thurston classification of mapping classes is determined by TQFT. J. Math. Kyoto Univ. 48 (2008), 323-338. MR 2436739 Zbl 1195.57065 
[4] J. E. Andersen, Mapping class groups do not have Kazhdan's Property (T). Preprint 2007 arXiv:0706.2184v1

[5] J. E. Andersen, N. L. Gammelgaard, and M. R. Lauridsen, Hitchin's connection in metaplectic quantization. Quantum Topol. 3 (2012), 327-357.

[6] J. E. Andersen, J. Mattes, and N. Yu. Reshetikhin, The Poisson structure on the moduli space of flat connections and chord diagrams. Topology 35 (1996), 1069-1083. MR 1404925 Zbl 0857.58009v1

[7] J. E. Andersen, J. Mattes and N. Yu. Reshetikhin, Quantization of the algebra of chord diagrams. Math. Proc. Cambridge Philos. Soc. 124 (1998), 451-467.

MR 1636568 Zbl 0943.57007

[8] J. E. Andersen and K. Ueno, Abelian conformal field theory and determinant bundles. Internat. J. Math. 18 (2007), 919-993. MR 2339577 Zbl 1128.81026

[9] J. E. Andersen and K. Ueno, Geometric construction of modular functors from conformal field theory. J. Knot Theory Ramifications 16 (2007), 127-202. MR 2306213 Zbl 1123.81041

[10] J. E. Andersen and K. Ueno, Modular functors are determined by their genus zero data. Quantum Topol. 3 (2012), 255-291.

[11] J. E. Andersen and K. Ueno, Construction of the Reshetikhin-Turaev TQFT from conformal field theory. Preprint 2011. arXiv:1110.5027v1

[12] J. E. Andersen and R. Villemoes, The first cohomology of the mapping class group with coefficients in algebraic functions on the $\mathrm{SL}_{2}(\mathbb{C})$ moduli space. Algebr. Geom. Topol. 9 (2009), 1177-1199. MR 2511142 Zbl 05567109

www.msp.warwick.ac.uk/agt/2009/09-02/p042.xhtml

[13] J. E. Andersen and R. Villemoes, Degree one cohomology with twisted coefficients of the mapping class group. In S. Akbulut et al. (eds.), Proceedings of the $17^{\text {th }}$ Gökova geometry-topology conference, Gökova, Turkey, May 31-June 4, 2010. International Press, Somerville (MA), and Gökova Geometry-Topology Conferences, Gökova, 2011, 64-78. Zbl 05994730

[14] J. E. Andersen and R. Villemoes, Cohomology of mapping class groups and the abelian moduli space. Quantum Topol. 3 (2012), 359-376.

[15] M. Atiyah, The Jones-Witten invariants of knots. Séminaire Bourbaki, Vol. 1989/90. Astérisque 189-190 (1990), Exp. No. 715, 7-16. MR 1099870 Zbl 0739.57001

[16] M. F. Atiyah and R. Bott, The Yang-Mills equations over Riemann surfaces. Philos. Trans. Roy. Soc. London Ser. A 308 (1982), 523-615. MR 0702806 Zbl 0509.14014

[17] S. Axelrod, S. Della Pietra, and E. Witten, Geometric quantization of Chern Simons gauge theory. J. Differential Geom. 33 (1991) 787-902. MR 1100212 Zbl 0697.53061

[18] A. L. Besse, Einstein manifolds. Ergebnisse der Mathematik und ihrer Grenzgebiete (3) 10. Springer Verlag, Berlin etc., 1987. MR 0867684 Zbl 0613.53001

[19] C. Blanchet, Hecke algebras, modular categories and 3-manifolds quantum invariants. Topology 39 (2000), 193-223. MR 1710999 Zbl 0938.57009

[20] C. Blanchet, N. Habegger, G. Masbaum, and P. Vogel, Three-manifold invariants derived from the Kauffman bracket. Topology 31 (1992), 685-699. MR 1191373 Zbl 0771.57004 
[21] C. Blanchet, N. Habegger, G. Masbaum, and P. Vogel, Topological quantum field theories derived from the Kauffman bracket. Topology 34 (1995), 883-927. MR 1362791 Zbl 0887.57009

[22] M. Bordeman, E. Meinrenken, and M. Schlichenmaier, Toeplitz quantization of Kähler manifolds and $g l(N), N \rightarrow \infty$ limit. Comm. Math. Phys. 165 (1994), 281-296. MR 1301849 Zbl 0813.58026 projecteuclid.org/getRecord?id=euclid.cmp/1104271132

[23] J.-M. Drezet and M. S. Narasimhan, Groupe de Picard des variétés de modules de fibrés semi-stables sur les courbes algébriques. Invent. Math. 97 (1989), 53-94. MR 0999313 Zbl 0689.14012

[24] G. Faltings, Stable G-bundles and projective connections. J. Algebraic Geom. 2 (1993), 507-568. MR 1211997 Zbl 0790.14019

[25] D. S. Freed, Classical Chern-Simons theory I. Adv. Math. 113 (1995), 237-303. MR 1337109 Zbl 0844.58039 www.sciencedirect.com/science/article/pii/S0001870885710390

[26] M. H. Freedman, K. Walker, and Z. Wang, Quantum SU(2) faithfully detects mapping class groups modulo center. Geom. Topol. 6 (2002), 523-539. MR 1943758

Zbl 1037.57024 www.msp.warwick.ac.uk/gt/2002/06/p018.xhtml

[27] V. V. Fock and A. A Rosly, Flat connections and polyubles. Teoret. Mat. Fiz. 95 (1993), 228-238. English trasl. in Theoret. and Math. Phys. 95 (1993), 526-534. MR 1243250 Zbl 0849.58030

[28] V. V. Fock and A. A Rosly, Moduli space of flat connections as a Poisson manifold. In Advances in quantum field theory and statistical mechanics: $2^{\text {nd }}$ Italian-Russian collaboration. Papers from the INTAS-Landau Network School held in Como, September 2-14, 1996. Internat. J. Modern Phys. B 11 (1997), 3195-3206. MR 1603053 Zbl 1229.58012

[29] B. van Geemen and A. J. de Jong, On Hitchin's connection. J. of Amer. Math. Soc. 11 (1998), 189-228. MR 1469656 Zbl 0920.32017

www.ams.org/journals/jams/1998-11-01/S0894-0347-98-00252-5/home.html

[30] S. Gutt and J. Rawnsley, Equivalence of star products on a symplectic manifold; an introduction to Deligne's Čech cohomology classes. J. of Geom. Phys. 29 (1999), 347-392. MR 1675581 Zbl 1024.53057

[31] N. J. Hitchin, Flat connections and geometric quantization. Comm. Math. Phys. 131 (1990), 347-380. MR 1065677 Zbl 0718.53021

projecteuclid.org/getRecord?id=euclid.cmp/1104200841

[32] A. V. Karabegov and M. Schlichenmaier, Identification of Berezin-Toeplitz deformation quantization. J. Reine Angew. Math. 540 (2001), 49-76. MR 1868597 Zbl 0997.53067

[33] Y. Laszlo, Hitchin's and WZW connections are the same. J. Diff. Geom. 49 (1998), 547576. MR 1669720 Zbl 0987.14027

[34] G. Masbaum, An element of infinite order in TQFT-representations of mapping class groups. In H. Nencka (ed.), Low dimensional topology. Proceedings of a conference, Funchal, Madeira, Portugal, January 12-17, 1998. Contemp. Math. 233. Amer. Math. Soc., Providence (RI), 1999. MR 1701678 Zbl 0929.57006

[35] G. Masbaum, Quantum representations of mapping class groups. In M. Boileau et al. (eds.), Groupes et Géométrie. SMF Journée annuelle 2003. Société Mathématique de France, Paris, 2003. 19-36. MR 2202283 Zbl 1078.57002 
[36] M. S. Narasimhan and C.S. Seshadri, Holomorphic vector bundles on a compact Riemann surface. Math. Ann. 155 (1964), 69 - 80. MR 0166799 Zbl 0122.16701

[37] M. S. Narasimhan and C. S. Seshadri, Stable and unitary vector bundles on a compact Riemann surface. Ann. Math. (2) 82 (1965), 540-67. MR0184252 Zbl 0171.04803

[38] T. R. Ramadas, Chern-Simons gauge theory and projectively flat vector bundles on Mg. Comm. Math. Phys. 128 (1990), 421-426. MR 1043527 Zbl 0706.53018 projecteuclid.org/getRecord?id=euclid.cmp/1104180437

[39] T. R. Ramadas, I. M. Singer, and J. Weitsman, Some Comments on Chern-Simons Gauge Theory. Comm. Math. Phys. 126 (1989), 409-420. MR 1027504 Zbl 0686.53066 projecteuclid.org/getRecord?id=euclid.cmp/1104179858

[40] N. Yu. Reshetikhin and V. G. Turaev, Ribbon graphs and their invariants derived from quantum groups. Comm. Math. Phys. 127 (1990), 1-26. MR 1036112 Zbl 0768.57003 projecteuclid.org/getRecord?id=euclid.cmp/1104180037

[41] N. Yu. Reshetikhin and V. G. Turaev, Invariants of 3-manifolds via link polynomials and quantum groups. Invent. Math. 103 (1991), 547-597. MR 1091619 Zbl 0725.57007

[42] J. Roberts, Irreducibility of some quantum representations of mapping class groups. $J$. Knot Theory and its Ramifications 10 (2001), 763-767. MR 1839700 Zbl 1001.57036

[43] P. Scheinost and M. Schottenloher, Metaplectic quantization of the moduli spaces of flat and parabolic bundles. J. Reine Angew. Math. 466 (1995), 145-219. MR 1353317 Zbl 0827.58010

[44] M. Schlichenmaier, Zwei Anwendungen algebraisch-geometrischer Methoden in der theoretischen Physik: Berezin-Toeplitz-Quantisierung und globale Algebren der zweidimensionalen konformen Feldtheorie. Habilitation thesis. University of Mannheim, Mannheim, 1996.

[45] M. Schlichenmaier, Deformation quantization of compact Kähler manifolds by BerezinToeplitz quantization. In G. Dito and D. Sternheimer (eds.), Conférence Moshé Flato 1999: Quantization, deformations, and symmetries, Dijon, France, September 5-8, 1999. Volume II. Mathematical Physics Studies 22. Kluwer Academic Publishers, Dordrecht, 2000, 289-306. MR 1805922 Zbl 1028.53085

[46] M. Schlichenmaier, Berezin-Toeplitz quantization and Berezin transform. In S. Graffi and A. Martinez, Long time behaviour of classical and quantum systems. Proceedings of the Bologna APTEX international conference, Bologna, Italy, September 13-17, 1999. Series on Concrete and Applicable Mathematics 1. World Scientific, Singapore, 2001, 271-287. MR 1852228 Zbl 0983.81035

[47] A. Tsuchiya, K. Ueno, and Y. Yamada, Conformal field theory on universal family of stable curves with gauge symmetries. In M. Jimbo et al. (eds), Integrable systems in quantum field theory and statistical mechanics. Based on papers presented at the symposia held at Research Institute for Mathematical Sciences, Kyoto University, Kyoto (Japan) during October 17-21, 1988, and at Kyuzeso, Katata (Japan) during October 24-28, 1988. Advanced Studies in Pure Mathematics 19. Academic Press, Boston (MA), 1989, 459-566. MR 1048605 Zbl 0696.17010

[48] V. G. Turaev, Quantum invariants of knots and 3-manifolds. De Gruyter Studies in Mathematics 18. Walter de Gruyter \& Co., Berlin, 1994. MR 1292673 Zbl 0812.57003 
Hitchin's connection, Toeplitz operators, and invariant deformation quantization 325

[49] G. M. Tuynman, Quantization: Towards a comparison between methods. J. Math. Phys. 28 (1987), 2829-2840. MR 0917637 Zbl 0639.58035

[50] E. Witten, Quantum field theory and the Jones polynomial. Comm. Math. Phys. 121 (1989), 351-399. MR 0990772 Zbl 0667.57005

projecteuclid.org/getRecord?id=euclid.cmp/1104178138

[51] N. M. J. Woodhouse, Geometric quantization. Second edition. Oxford Mathematical Monographs. Oxford Science Publications. The Clarendon Press, Oxford University Press, New York, 1992. MR 1183739 Zbl 0747.58004

Received February 24, 2010

Jørgen Ellegaard Andersen, Center for Quantum Geometry of Moduli Spaces, University of Aarhus, 8000 Aarhus, Denmark

E-mail: andersen@qgm.au.dk 